1 A population genomic unveiling of a new cryptic mosquito taxon within the malaria-

2 transmitting Anopheles gambiae complex

4 Jacob A. Tennessen ${ }^{1,2, *}$, Victoria A. Ingham ${ }^{3}$, Kobié Hyacinthe Toé4, Wamdaogo Moussa

5 Guelbéogo $^{4}, \mathrm{~N}^{\text {’Falé Sagnon }}{ }^{4}$, Rebecca Kuzma ${ }^{1,2}$, Hilary Ranson ${ }^{3}$, Daniel E. Neafsey ${ }^{1,2}$

6

$7 \quad{ }^{1}$ Harvard T.H. Chan School of Public Health, Boston, MA USA

$8 \quad{ }^{2}$ Broad Institute, Cambridge, MA USA

$9 \quad{ }^{3}$ Liverpool School of Tropical Medicine, Liverpool, UK

$10{ }^{4}$ Centre National de Recherche et de Formation sur le Paludisme, Ouagadougou, Burkina

11 Faso

12 *corresponding author

14 Running title: A new cryptic taxon of Anopheles mosquito

16 Abstract

18 The Anopheles gambiae complex consists of multiple morphologically indistinguishable 19 mosquito species including the most important vectors of the malaria parasite Plasmodium

20 falciparum in sub-Saharan Africa. Nine cryptic species have been described so far within the 21 complex. The ecological, immunological, and reproductive differences among these species 22 will critically impact population responses to disease control strategies and environmental 23 changes. Here we examine whole-genome sequencing data from a longitudinal study of 
24 putative $A$. coluzzii in western Burkina Faso. Surprisingly, many specimens are genetically

25 divergent from A. coluzzii and all other Anopheles species and represent a new taxon, here

26 designated Anopheles TENGRELA (AT). Population genetic analysis suggests that the

27 cryptic GOUNDRY subgroup, previously collected as larvae in central Burkina Faso, 28 represents an admixed population descended from both $A$. coluzzii and AT. AT harbors low 29 nucleotide diversity except for the $2 \mathrm{La}$ inversion polymorphism which is maintained by 30 overdominance. It shows numerous fixed differences with $A$. coluzzii concentrated in several 31 regions reflecting selective sweeps, but the two taxa are identical at standard diagnostic loci 32 used for taxon identification and thus AT may often go unnoticed. We present an amplicon33 based genotyping assay for identifying AT which could be usefully applied to numerous 34 existing samples. Misidentified cryptic taxa could seriously confound ongoing studies of about evolutionary dynamics of Anopheles and Plasmodium.

Keywords: Anopheles, vector, cryptic taxa, admixture, selective sweep, reproductive barrier

Introduction 
47 parasites (Miller, 2002; WHO, 2018). Mosquito-targeting interventions are by far the most

48 effective at reducing malaria infection, substantially exceeding the impact of those that target the

49 parasite directly, like artemisinin combination therapy (Bhatt et al., 2016). However, evolutionary

50 processes such as selection for resistance to insecticides have repeatedly allowed mosquitoes to

51 evade control efforts (Ranson \& Lissenden, 2016). Similarly, adaptive resistance is likely to

52 frustrate new control technologies such as gene drives, which involve manipulating mosquito

53 evolution directly (Marshall et al., 2019). Control strategies will need to anticipate and foil these

54 adaptive responses and thus can only succeed if Anopheles population genetics is thoroughly

55 understood.

56 In sub-Saharan Africa, the most important definitive hosts for Plasmodium are mosquitoes

57 of the Anopheles gambiae species complex. These mosquitoes are among the most genetically

58 diverse animals on Earth (Leffler et al., 2012; Anopheles gambiae 1000 Genomes Consortium,

59 2017). The clade contains nine morphologically-identical species, three of which were only

60 described in the last decade (Coetzee et al., 2013; Barrón et al., 2019). The evolutionary

61 relationships among these cryptic species are complicated due to incomplete lineage sorting and

62 introgression facilitated by porous reproductive barriers (Fontaine et al., 2015). A majority of the

63 genome can cross species boundaries, and this is a frequent and recent phenomenon in response to

64 novel selective pressures such as insecticides (Clarkson et al., 2014; Main et al., 2015; Norris et

65 al., 2015). Additionally, the taxonomic status of some rare yet distinct groups within this complex

66 remains unclear. The subgroup GOUNDRY was identified in Burkina Faso and found to be

67 genetically distinct from its closest known relative, A. coluzzii (Riehle et al., 2011; Crawford et

68 al., 2015; Crawford et al., 2016). While GOUNDRY has not been formally described as a separate

69 species, it is genetically distinct from any known species. Further characterization has been 
70 impeded because only larval stages have been collected in the field and collecting additional

71 GOUNDRY individuals has proven difficult. There is a substantial need to better understand

72 patterns of gene flow and partitioning of genetic diversity within the A. gambiae complex, in order

73 to better predict and mitigate the inevitable evolutionary counterstrategies to vector control efforts.

$74 \quad$ In this paper, we use whole genome sequencing data to identify yet another new cryptic

75 taxon within the A. gambiae complex, occurring in a country (Burkina Faso) where many previous

76 surveys of anopheline mosquitoes have occurred. Though this taxon is closely related to $A$.

77 coluzzii, it shows substantial yet incomplete reproductive incompatibility with it. This new taxon,

78 Anopheles TENGRELA (AT), clarifies the origin of GOUNDRY and illuminates the complicated

79 interplay between migration and isolation that characterizes these mosquitoes.

80

81 Materials and Methods

$82 \quad$ Samples and sequencing

83 We chose 287 specimens of putative A. coluzzii from larval collections in Tengrela,

84 Burkina Faso $\left(10.7^{\circ} \mathrm{N}, 4.8^{\circ} \mathrm{W}\right)$ (Table 1). All specimens were reared to adults and typed as $A$.

85 coluzzii females based on morphology (Gillies \& De Meillon, 1968) and standard molecular assays

86 (Santolamazza et al, 2008). They comprised a longitudinal series across four years (2011, 2012,

87 2015, and 2016) which were examined as part of a study on insecticide resistance evolution. We

88 extracted DNA from individual mosquitoes using a Qiagen DNeasy Blood \& Tissue Kit (Qiagen)

89 following manufacturer's instructions. We sequenced whole genomic DNA with 151 bp paired-

90 end reads on an Illumina HiSeq $\mathrm{X}$ instrument at the Broad Institute, using Nextera low-input

91 sequencing libraries. 
Identification of AT

All reads were aligned to the Anopheles gambiae PEST reference genome (assembly AgamP4; Holt et al., 2002; Sharakhova et al., 2007) using bwamem v. 0.7.17 (Li \& Durbin, 2009; command: bwa mem -M) and samtools v .1.8 (Li 2011; commands: samtools view -h -F 4 -b,

97 samtools sort, samtools index) and variants were called using GATK v. 3.8-1 (McKenna et al., 98 2010; hard filtering of single nucleotide polymorphisms (SNPs) with QD $<5$ and/or FS $>60$, and 99 indels with QD $<2$ and/or FS > 200; --max-gaussians 4). Initial analysis was restricted to 100 individuals with at least $8 \mathrm{x}$ median coverage, with variants filtered to have at least $8 \mathrm{x}$ coverage in 101 all individuals, be at least 500 bp apart, and not show heterozygote excess in violation of Hardy102 Weinberg expectations. With this dataset, AT was identified as distinct from A. coluzzii using 103 principal component analysis (PCA) with the princomp function in R (v. 3.6.2; R Core Team, 104 2019). Lower-coverage individuals were subsequently designated as AT or A. coluzzii based on 105 markers that differentiate these taxa in the high-coverage individuals; eight individuals (coverage $1060-2 x$ ) could not be unambiguously assigned to taxon and were subsequently ignored, leaving 279 107 acceptable individuals.

108 Standard statistical tests and data visualization were performed in R (v. 3.6.2; R Core Team 109 2019). Phylogenetic analysis employed RAxML with -m GTRCAT (Stamatakis 2006). Anopheles 110 genomes used in phylogenetic analysis (other than AT and A. coluzzii from Tengrela) were: $A$. 111 coluzzii from Burkina Faso at locations (Bana, Pala, and Souroukoudinga) approximately 112 intermediate between Tengrela and the GOUNDRY sites (ERS224009, ERS224023, ERS223804, 113 ERS223963, ERS224782, ERS223946), A. gambiae (PEST reference genome, ERS223759, 
114 ERS224149, ERS223976, ERS224154, ERS224132, and ERS224151) A. arabiensis

115 (SRR3715623 and SRR3715622), A. quadriannulatus (SRR1055286 and SRR1508190), A.

116 bwambae (SRR1255391 and SRR1255390), A. melas (SRR561803 and SRR606147), and $A$.

117 merus (SRR1055284). Annotation and analysis of key genomic regions and alleles was facilitated

118 by VectorBase (Giraldo-Calderón et al., 2015) and the Ag1000G genomic resources (Anopheles

119 gambiae 1000 Genomes Consortium, 2017).

In order to directly compare our specimens with GOUNDRY, we examined the previously

123 published whole-genome sequences of GOUNDRY and A. coluzzii (Crawford et al., 2016;

124 BioProject PRJNA273873). We then examined a representative dataset of 51 AT genomes, $51 A$.

125 coluzzii genomes from Tengrela, 12 GOUNDRY genomes, and 10 A. coluzzii genomes from

126 GOUNDRY habitats (Kodougou and Goundry). To minimize artifacts due to read alignment, we

127 trimmed our Tengrela reads to $100 \mathrm{bp}$ to match the GOUNDRY data, and then aligned all reads to

128 the PEST reference genome using bwamem and samtools as above and called genotypes jointly

129 using bcftools v. 1.8 (Li 2011; commands: bcftools mpileup, bcftools call -m -Ov -v). For 130 demographic analysis, we removed any variants with missing genotypes, as above we filtered

131 variants to be at least $500 \mathrm{bp}$ apart and to not show heterozygote excess in violation of Hardy-

132 Weinberg expectations, we excluded sites in the 2La inversion, and we separated this jointly-called 133 and filtered dataset into autosomes (84,550 sites) and X chromosome (9,212 sites).

134 We used this jointly-called dataset for demographic analysis with dadi (Gutenkunsk, 135 Hernandez, Williamson, \& Bustamante, 2009). Our goal was to generate a plausible demographic 
model for these populations and in particular to test whether GOUNDRY derives from an

137 admixture event between AT and A. coluzzii. We ran multiple models, including models without

138 migration, without population size changes, or with only two periods of differing demographic

139 parameters. Critically for this analysis, we ran models identical to the preferred model but with

140 GOUNDRY originating entirely from either AT or A. coluzzii; rejecting these models thus

141 demonstrates that GOUNDRY is admixed (Supp Table 1). These models estimate multiple

142 parameters, the absolute values of which depend upon numerous assumptions that are challenging

143 to validate. For example, heterozygosity per bp in our full dataset is 400x higher than in the filtered

144 dataset because most variants are filtered, and we estimate that the full dataset represents $85 \%$ of

145 the genome, with the rest occurring in long stretches (over $1 \mathrm{~kb}$ ) without called genotypes that may

146 be inaccessible to our genotyping pipeline; thus we adjusted our estimate of nucleotide diversity

147 upwards accordingly when inferring effective population size. However, this estimate is likely

148 imprecise, as low-quality sites that were filtered away could overestimate heterozygosity if

149 enriched for mismatched reads, or could underestimate heterozygosity if divergent reads failed to

150 align to the reference genome, Similarly, we assumed a mutation rate of $3 \times 10^{-9}$ (Keightley, Ness,

151 Halligan, \& Haddrill, 2014), and ten generations per year. In contrast to absolute values of these

152 point estimates, the relative values among parameters (e.g. population size of AT versus $A$.

153 coluzzii), and among model likelihoods, should be more robust. As above, we examined autosomal

154 genotypes and $\mathrm{X}$ chromosome genotypes separately. To assess the robustness of our results, we

155 ran dadi on datasets with different levels of filtration, such that the minimum distance among

156 variants was $100,200,500$, or $1000 \mathrm{bp}$; as with other analyses we present the $500 \mathrm{bp}$ threshold as

157 the default. 
We confirmed the demographic results from dadi using the same jointly-called dataset in

159 other analyses. We conducted PCA as above. We also conducted an ADMIXTURE analysis

160 (Alexander, Novembre, \& Lange, 2009) with K ranging from 1 to 5, choosing the value of K with

161 the lowest cross-validation error as recommended. Finally, we used TreeMix (Pickrell \& Pritchard

162 2012) to generate a phylogeny among populations and test for migration. We first incorporated

163 genotypes from 115 specimens of A. gambiae from Ag1000G, yielding a dataset with 49,645

164 autosomal and $997 \mathrm{X}$ chromosome variants. We created a phylogeny with the possibility of

165 migration (treemix function, -m 1 or $-\mathrm{m} 2$, -root $A$. gambiae). We used the threepop and fourpop

166 functions (-k 100) to calculate $f_{3}$ and $f_{4}$.

Genomic characterization of AT

Across the genome, population genetic statistics such as F FT, $\pi$, Tajima's D, and Dxy were

170 calculated with Perl scripts incorporating the Perlymorphism scripts (Bio::PopGen, BioPerl

171 version 1.007000; Stajich \& Hahn, 2005). FST calculations followed the script FstPerSite.pl

172 available at https://github.com/jacobtennessen/MalariaHallmarks/. Statistics were examined in

173 overlapping sliding windows of $1 \mathrm{Mb}$ or $100 \mathrm{~kb}$. We identified putative selective sweep regions

174 as those showing Tajima's D under -2.5 and $\pi$ under 0.0005 . We considered "definitive

175 differences" (i.e. fixed or nearly fixed alleles) between AT and A. coluzzii to be sites showing FST

$176>0.99$. At several notable loci we directly compared genotypes in AT, GOUNDRY, and A. coluzzii

177 (Table 2), including the intergenic region (IGS) of rRNA (Scott, Brogdon, \& Collins, 1993;

178 Fanello, Santolamazza, \& della Torre, 2002), indels in SINE200 retrotransposon S200 X6.1

179 (Santolamazza et al., 2008), and "divergence island SNPs" showing nearly-fixed differences 
among previously described taxa (Lee et al., 2013). We identified numerous variants spanning over 20 Mb from 2L_20569357 to 2L_42087028 showing perfect linkage disequilibrium with each

182 other in our data; we inferred that these variants represent the $2 \mathrm{La}$ inversion and used a set of a 70 183 such variants, all more than $1 \mathrm{~kb}$ apart, to genotype $2 \mathrm{La}$ in all individuals. Although all specimens 184 were phenotyped as female, we examined coverage across the $\mathrm{X}$ and $\mathrm{Y}$ chromosomes in the 185 reference genome in order to infer sex chromosome karyotype. $\mathrm{X}$ chromosome coverage 186 approximately equal to autosomal coverage was interpreted as XX karyotype. Y chromosome 187 coverage equal to or greater to autosomal coverage across the majority of the Y chromosome was 188 interpreted to mean that sequence which is male-specific in PEST occurs in our female specimens. 189 To infer whether such sequence could constitute a functional $\mathrm{Y}$ chromosome, we assessed 190 coverage at sex-determining gene $Y G 2$.

191 We used several tactics to test for inbreeding and its potential effects. For all analyses 192 including those mentioned above, we filtered out sites showing heterozygote excess in violation 193 of Hardy-Weinberg equilibrium but not sites showing homozygote excess, since the former are 194 more likely to be caused by alignment errors and the latter could be biologically real due to 195 population structure. This filtering strategy could only bias the dataset toward excess 196 homozygosity. To calculate the F coefficient, we used the -het function in PLINK (v1.90b3.32, 197 Chang et al. 2015) treating AT, A. coluzzii, and GOUNDRY separately. A positive F coefficient 198 is indicative of inbreeding. For all individuals, we calculated heterozygosity $(H)$ in $1 \mathrm{Mb}$ windows 199 across the genome and looked for homozygosity tracts showing $H \leq 1$ e-06 (i.e. no more than one 200 heterozygous polymorphism per $\mathrm{Mb}$ ). To assess whether homozygosity tracts are genomic outliers 201 or consistent with genome-wide levels of polymorphism, we examined the distribution of 202 heterozygosity per genomic window per individual. We counted homozygous doubletons (i.e. 
203 homozygotes for an allele otherwise absent in the population) and tested whether these are enriched

204 in homozygosity tracts. Finally, to test whether inbreeding would be likely to influence our results,

205 we generated a perfectly homozygous set of autosomes in silico by selecting only a single allele

206 per individual at all autosomal sites. Using this haploid dataset, we replicated our ADMIXTURE

207 and dadi analyses.

\section{Amplicon genotyping}

We designed and tested an amplicon-based genotyping method to identify AT in additional

211 samples. We selected 50 diagnostic SNPs and small indels, each with a non-reference allele that

212 is fixed in all AT specimens but absent in Tengrela A. coluzzii and the Ag1000G data (Anopheles

213 gambiae 1000 Genomes Consortium, 2017). For each of these, we designed a primer pair to

214 amplify a PCR product 160 to 230 bp in size using BatchPrimer3 (You et al., 2008). We ensured

215 that primers did not overlap common polymorphisms found in A. coluzzii or A. gambiae

216 (Anopheles gambiae 1000 Genomes Consortium, 2017). We ordered primers with the Nextera

217 Transposase Adapters sequences added to the $5^{\prime}$ end in order to eliminate the initial ligation step

218 in library preparation (primer 1: $5^{\prime}$ TCGTCGGCAGCGTCAGATGTGTATAAGAGACAG-

219 [locus specific sequence]; primer 2: 5' GTCTCGTGGGCTCGGAGATGTGTATAAGAGACAG-

220 [locus specific sequence]).

221 Primers were tested individually and in various pools of primer pairs to amplify jointly in 222 multiplex PCR using known samples of AT, A. coluzzii, and A. gambiae. We PCR-amplified 2ng 223 of each DNA sample using the Veriti 96-well Fast Thermal Cycler (Applied Biosystems, Waltham, 224 Massachusetts) with 12.5uL (62.5 U) of Multiplex PCR Master Mix (Qiagen, Hilden, Germany), 
$2.5 \mathrm{uL}$ of the pre-mixed primer pool $(200 \mathrm{nM})$, and $8 \mathrm{uL}$ of nuclease-free water. The PCR

226 conditions were as follows: $95^{\circ} \mathrm{C}$ for 15 minutes; 30 cycles of $94{ }^{\circ} \mathrm{C}$ for 30 seconds and $60{ }^{\circ} \mathrm{C}$ for

22790 seconds, $72{ }^{\circ} \mathrm{C}$ for 90 seconds; and $72{ }^{\circ} \mathrm{C}$ for 10 minutes. Initial confirmation of correctly sized

228 amplicons was done by gel electrophoresis.

We performed a second round of PCR to attach i5 and i7 Illumina indices (Nextera XT

230 Index Kit v2 set A; Illumina, San Diego, California) to the PCR amplicons. The second round PCR

231 reaction included 5 uL of 1X Platinum SuperFi Library Amplification Master Mix (Thermo Fisher,

232 Waltham, Massachusetts), $5 \mathrm{uL}$ of the first round PCR product, and $1 \mathrm{uL}$ of premixed i5/i7 index

233 primers. The PCR conditions were as follows: $98{ }^{\circ} \mathrm{C}$ for 30 seconds; 10 cycles of $98{ }^{\circ} \mathrm{C}$ for 15

234 seconds, $60^{\circ} \mathrm{C}$ for 30 seconds, and $72{ }^{\circ} \mathrm{C}$ for 30 seconds; and $72{ }^{\circ} \mathrm{C}$ for 1 minute. Confirmation of

235 amplification was done by gel electrophoresis.

Amplicon libraries were purified using Agencourt AMPure XP beads (Beckman Coulter,

237 Brea, California) at $1.8 \mathrm{x}$ with a final elution volume of $35 \mathrm{uL}$. Library concentrations were

238 determined using Qubit HS DNA kit (Thermo Fisher, Waltham, Massachusetts). Library

239 concentration and size was confirmed using the Agilent 2100 Bioanalyzer instrument. Libraries

240 were pooled in equimolar concentration and diluted to 180 pmol. A $15 \%$ PhiX spike in was added

241 to the diluted pool. We loaded $20 \mathrm{uL}$ of the final pool onto an Illumina iSeq 100 instrument at the

242 Broad Institute per the manufacturer's protocol and sequenced these with $151 \mathrm{bp}$ paired-end reads.

243 Genotype could be inferred from the resultant reads without an alignment step, simply by

244 counting reads containing diagnostic kmers (24-33bp) overlapping the target polymorphism. After

245 determining that a pool of 5 primer pairs sequenced by iSeq was sufficient for taxon identification,

246 we used it to amplify and sequence a novel set of 79 putative A. coluzzii larvae (from either near 
247 Tengrela in southwestern Burkina Faso or else of unknown origin; Table 1), alongside a known

248 Tengrela A. coluzzii control and 16 empty well negative controls.

250 Results

A new cryptically isolated lineage

In whole-genome sequencing data of 279 putative $A$. coluzzii specimens from Tengrela,

254 Burkina Faso (median coverage = 16x; dataset Tennessen et al., 2020), collected across four years,

25551 specimens were unexpectedly distinct (Figure 1A). This signal is robust across both autosomes

256 and the $\mathrm{X}$ chromosome, and for more stringent filtering approaches (Supp Figure 1). The divergent

257 individuals, here designated Anopheles TENGRELA (AT), were all collected as larvae in 2011,

258 the only year in which sample collection included temporary puddles in addition to rice paddies.

259 Most (72\%) specimens from 2011 were AT. AT is not a close match to any known sequenced 260 samples of A. coluzzii or A. gambiae (Anopheles gambiae 1000 Genomes Consortium, 2017). It is

261 similar but not identical to GOUNDRY, which was also described from temporary puddles in

262 Burkina Faso, from sites 250-550 km from Tengrela (Riehle et al., 2011). The genetic divergence

263 from sympatric $A$. coluzzii individuals collected simultaneously suggests a strong reproductive 264 barrier.

To further investigate the distinction between AT and GOUNDRY, we jointly analyzed

266 our Tengrela data alongside whole genome sequence data from GOUNDRY and sympatric $A$.

267 coluzzii specimens (Crawford et al., 2016). To account for differences in sequencing platforms and

268 genotyping algorithms, we trimmed all reads to $100 \mathrm{bp}$ and jointly aligned them and called

269 genotypes. While $A$. coluzzii from Tengrela and $A$. coluzzii from the GOUNDRY sites were 
270 genetically indistinguishable, AT and GOUNDRY remained distinct from each other (Figure 1B).

271 Thus, the distinctiveness of AT is not owing to genotyping artifact, and the divergence between

272 AT and GOUNDRY is greater than that seen for A. coluzzii populations over the same physical

273 distance. We therefore infer that AT is not simply an additional population of GOUNDRY.

274 To examine the relationship between AT and the broader A. gambiae species complex, we

275 divided the genome into $100 \mathrm{~kb}$ windows and ran a phylogenetic analysis with seven nominal

276 species (Figure 2A). The AT genome is typically sister to A. coluzzii (42.1\% of windows), A.

277 gambiae (33.1\% of windows), or to a clade containing only A. coluzzii and A. gambiae (20.4\% of

278 windows), with only $4.5 \%$ of windows displaying an alternate topology (Supp Figure 2). The

279 relationship between AT and A. coluzzii is especially strong on the X chromosome (Figure 2A;

280 Supp Figure 2), which in the A. gambiae complex is thought to reflect phylogenetic signal more

281 accurately than the autosomes (Fontaine et al., 2015). As A. coluzzii is most often the closest

282 species, and much of the similarity to A. gambiae is owing to the 2La inversion (Supp Figure 2),

283 we consider A. coluzzii to be the sister taxon to AT for all subsequent analyses.

284 We constructed a mitochondrial phylogeny of AT, GOUNDRY, and representative

285 samples of A. coluzzii, A. gambiae, and A. arabiensis (Figure 2B). All AT individuals share the

286 same haplotype, which does not occur in any other taxon except for a single GOUNDRY

287 individual. GOUNDRY samples occur in two clades, the one containing AT and another one

288 nested within A. coluzzii and A. gambiae, consistent with maternal ancestry from both lineages.

291 GOUNDRY derives from AT and A. coluzzii 
GOUNDRY is closely related to AT, but genetically distinct (median FsT $=0.11$; mean FsT

$293=0.12$; Supp Figure 3). GOUNDRY also shows substantial recent ancestry from A. coluzzii..

294 Several independent analyses, described below, support the conclusion that GOUNDRY 295 autosomes are admixed with 15-20\% A. coluzzii ancestry, while GOUNDRY X chromosomes are 296 almost entirely AT-like. There are relatively few fixed differences between GOUNDRY and AT, 297 but several occur on all chromosomes including the X (Supp Figure 3).

Analysis of AT, A. coluzzii from Burkina Faso, and GOUNDRY autosomes with 299 ADMIXTURE (Alexander et al., 2009) suggests two ancestral populations (K=2; Figure 3A). 300 Populations 1 and 2 are closely approximated by the contemporary AT and A. coluzzii populations, 301 respectively (AT: all individuals have $100.0 \%$ population 1 ancestry; A. coluzzii: mean population 3021 ancestry $=0.1 \%$, range $=0-7.4 \%)$. GOUNDRY is admixed from both populations, with a 303 majority of ancestry from AT (mean population 1 ancestry $=84.6 \%$, range $=76.6-88.7 \%$ ). The $\mathrm{X}$ 304 chromosome also suggests a two-population model differentiating contemporary AT and $A$. 305 coluzzii, but it assigns GOUNDRY nearly complete population 1 (AT-like) ancestry (mean = $30699.6 \%$, range $=97.1-100 \%$.

307 We confirmed the signal of admixture by fitting demographic models to the two308 dimensional site frequency spectrum with dadi (Gutenkunst et al., 2009). In our best-fitting 309 autosomal model, the lineages of A. coluzzii and AT diverged 1.7 million generations ago, 310 maintained a small degree of continuous gene flow, and then hybridized less than 10,000 311 generations ago to form GOUNDRY (Figure 3B; Supp Figure 4; Supp Table 1). Our model 312 included three different time periods among which population sizes and migration rates were 313 allowed to vary. Consistent with the relatively low nucleotide diversity, the effective population 314 size $(N)$ of AT was much smaller than A. coluzzii across all time periods. It was approximately 
200-fold smaller in the earliest and longest period, expanded during the middle time period, and

316 contracted again to be more than 10,000 -fold smaller than A. coluzzii today $\left(3.1 \times 10^{4}\right.$ and $3.6 \times 10^{8}$,

317 respectively). Migration rates $(\mathrm{m})$ ranged from $10^{-8}$ to $10^{-5}$, such that the effective number of

318 migrants per generation ( $m$ times $N$ of the recipient population) has only sporadically been high

319 enough to overcome the effects of $\operatorname{drift}(\mathrm{Nm}>1)$. In particular, gene flow from A. coluzzi into AT

320 has only been non-negligible during the middle period 41,000-967,000 generations ago, when the

321 AT population size was large and migration from A. coluzzii was substantial. GOUNDRY is

322 admixed with $81.0 \%$ ancestry from AT and $19.0 \%$ ancestry from A. coluzzii, with $N$ slightly larger

323 than AT $\left(4.1 \times 10^{4}\right)$. All four filtration strategies for autosomal variants support the same overall

324 scenario, with varying parameter estimates: the effective sizes of modern AT and GOUNDRY are

325134 to 20,225 times smaller than A. coluzzii, AT has decreased in population size while A. coluzzii

326 has increased, GOUNDRY is admixed with the proportion of A. coluzzii ancestry ranging from

$32713.7 \%$ to $30.7 \%$, and the split between AT and A. coluzzii is 99 to 636 times older than the origin

328 of GOUNDRY. In contrast, the best model for X chromosome data has GOUNDRY derived

329 entirely from the AT branch without gene flow from A. coluzzii (Supp Table 1).

330 We further investigated the signal of admixture with TreeMix (Pickrell \& Pritchard 2012),

331 incorporating A. gambiae alongside A. coluzzii, AT, and GOUNDRY. Autosomal data suggest a

332 tree with GOUNDRY sister to AT, but with relatively weak migration (weight $=0.10$ ) from $A$.

333 coluzzii to GOUNDRY (Figure 3C), and no support for any second migration event elsewhere in

334 the tree. As corroboration, $f_{4}$ is nonzero at $1.9 \mathrm{e}-04(\mathrm{p}<0.05)$, indicating a significant but relatively

335 weak signal of gene flow. However, $f_{3}$ is positive for any of the four populations compared with

336 any other two, meaning that this test provides no evidence for admixture. The $\mathrm{X}$ chromosome

337 shows no evidence of GOUNDRY admixture, neither upon the phylogeny nor with $f_{4}$ or $f_{3}$. 
Mosquitoes in the A. gambiae complex are typically identified with established molecular

341 markers. Analysis of these regions in AT reveals how this taxon easily goes undetected, both in

342 our initial survey of these samples and possibly in other studies (Table 2). At IGS, AT reliably

343 lacks the HhaI restriction site found in A. gambiae s. s., and instead harbors the AT dinucleotide

344 typical of $A$. coluzzii (Fanello et al., 2002). At S200 X6.1, AT possesses the 230 bp insertion

345 characteristic of $A$. coluzzii (Santolamazza et al., 2008). Interestingly, a SNP within this indel is

346 nearly fixed between AT and A. coluzzii, suggesting that amplification of this region could still be

347 diagnostic if it were sequenced and not merely assessed for band size. At divergence island SNPs,

348 AT appears identical to Tengrela A. coluzzii, although such SNPs on chromosome 2L are 349 polymorphic in both taxa.

350 In AT both haplotypes of the 2La inversion are common, unlike in A. coluzzii from 351 Tengrela or elsewhere (Coluzzi et al., 2002; Neafsey et al., 2010), and thus this $22 \mathrm{Mb}$ region 352 represents one of its most striking differences from A. coluzzii (Figure 4A). The $2 \mathrm{~L}+{ }^{\mathrm{a}}$ haplotype 353 occurs at a frequency of $48 \%$ and shows a remarkable heterozygote excess in violation of Hardy354 Weinberg expectations (expected heterozygotes $=25.4$, observed heterozygotes $=37 ; \chi^{2}=10.5 ; P$ $355=0.001)$, suggesting at least half of homozygous genotypes are selected against. This observation 356 is unexpected, since either the $2 \mathrm{~L}^{\mathrm{a}}$ or $2 \mathrm{~L}+{ }^{\mathrm{a}}$ haplotype can be the major allele across Anopheles 357 populations, and thus the inversion is thought to be maintained by geographically-varying selection 358 rather than heterozygote advantage (White et al., 2007). In contrast to AT, GOUNDRY 2La is in 359 Hardy-Weinberg equilibrium (Riehle et al. 2011). Our results suggest that the genomic background 360 of AT may facilitate overdominance at this inversion, and thus AT may be an important genetic 
reservoir which helps to maintain the polymorphism across the genus. Genes potentially under

362 selection in $2 \mathrm{La}$ include the highly polymorphic APL1 gene complex, implicated in immunity 363 against Plasmodium (Rottschaefer et al., 2011), and $R d l$, at which a nonsynonymous Ala-Ser 364 variant conveys resistance to the insecticide dieldrin (Du et sl. 2005). In APL1, the protective 365 APL1A ${ }^{2}$ haplotype is rare in AT (15\%) but is the major allele in Tengrela A. coluzzii (80\%). At $366 R d l$, resistant Ser occurs at $32 \%$ frequency in AT. In Tengrela A. coluzzii, this variant represents 367 the largest shift in allele frequency across years (Supp Figure 5), decreasing from 68\% in 20113682012 to $38 \%$ in 2015-2016, consistent for selection against costly resistance as organochloride use 369 has declined. While $R d l$ in A. coluzzii must occur on the (nearly fixed) $2 \mathrm{~L}^{\mathrm{a}}$ haplotype, in AT it is 370 more often associated with $2 \mathrm{~L}+^{\mathrm{a}}$.

371 Several other genomic regions are unusually divergent between AT and A. coluzzii (Table 372 2). Along with $2 \mathrm{La}$, both $T E P 1$ and $C Y P 9 K 1$ are outliers with respect to mean Fst between these 373 taxa (Figure 4A). TEPI encodes a complement-like immunity protein that occurs in highly 374 dissimilar allelic forms correlated with resistance to Plasmodium (White et al., 2011). All TEP1 375 alleles in AT are of the S (susceptible) type, while the R (resistant) type is nearly fixed in Tengrela 376 A. coluzzii. CYP9K1 is a P450 gene associated with resistance to insecticide (Main et al., 2015). 377 The cyp-II haplotype of the CYP9K1 region is fixed in AT and rare in Tengrela A. coluzzii; there 378 is suggestive but inconclusive evidence that this allele conveys increased resistance (Main et al., 379 2015). In contrast to the pronounced divergence at $C Y P 9 K 1$, the well-known insecticide-resistance 380 polymorphism $K d r$ (Donnelly et al., 2009) shows similar, intermediate frequencies across both AT 381 and Tengrela A. coluzzii. Nucleotide diversity is substantially lower in AT $(\pi=0.007)$ than in A. coluzzii $(\pi=0.012)$ 383 (Figure 4B). This trend is consistent across the genome except within the 2La inversion (AT: $\pi=$ 
3840.015 in 2La, $\pi=0.006$ elsewhere; A. coluzzii: $\pi=0.013$ in $2 \mathrm{La}, \pi=0.012$ elsewhere). This 385 difference is also consistent among individuals, such that the range of heterozygosity per 386 individual for AT does not overlap that of A. coluzzii (Supp Figure 6). Across the genome, 387 divergence between these taxa as measured by Dxy closely matches $\pi$ in $A$. coluzzii, as this taxon 388 that contributes the majority of variation; the exception occurs in 2La, when both Dxy and AT $\pi$ 389 are maximized. The site frequency spectra of AT and A. coluzzii are also quite distinct. In A. 390 coluzzii, Tajima's $\mathrm{D}$ is very negative $(\mathrm{D}=-2.0)$ and fairly uniform across the genome $(\mathrm{SD}=0.2)$, 391 reflecting its recent population expansion (Figure 3B; Anopheles gambiae 1000 Genomes 392 Consortium, 2017). In contrast, Tajima's D in AT is positive on average $(\mathrm{D}=1.3)$, consistent with 393 a recent dramatic decrease in population size (Figure 3B), but it's also quite variable across the 394 genome $(\mathrm{SD}=1.3)$. Three genomic regions in AT, comprising about $2 \%$ of the genome, show a 395 combination of highly negative Tajima's D and unusually low nucleotide diversity, and together 396 they harbor a third of the "definitive differences" (defined here as FST > 0.99; Figure 4A) between 397 AT and A. coluzzii. This suite of signals suggests positive selective sweeps in these three regions 398 in AT (Figure 4B). One putative sweep is observed on 3R. Though the signal extends from 399 approximately $8.9 \mathrm{Mb}$ to $13.3 \mathrm{Mb}$, it is concentrated in a one-megabase section from 11.5 to 12.5 $400 \mathrm{Mb}$ which contains 125 definitive differences (7\% of the genome-wide total) and also the lowest 401 autosomal nucleotide diversity $(\pi=0.0003)$ and lowest Tajima's $\mathrm{D}$ genome-wide $(\mathrm{D}=-2.9)$. 402 Multiple genes occur in or near this region, with no obvious single selection target. Several of 403 these genes have known phenotypic effects, including IR21a which encodes a thermoreceptor 404 implicated in heat-mediated host-seeking (Greppi et al., 2020), and a cluster of cuticular proteins 405 tied to insecticide resistance (Nkya et al., 2014; Huang et al., 2018). The other two selective sweep 406 signals occur on the $\mathrm{X}$ chromosome, which even outside of these regions shows lower nucleotide 
407 diversity overall than the autosomes. The putative inversion $\mathrm{Xh}$ between 8.5 to $10.0 \mathrm{Mb}$, which

408 also shows a sweep signal in GOUNDRY (Crawford et al., 2016), contains $15 \%$ of all definitive

409 differences with A. coluzzii and has the lowest nucleotide diversity in the AT genome $(\pi=0.0001)$.

410 The third signal overlaps CYP9K1 on the $\mathrm{X}$ between 13.5 to $16.0 \mathrm{Mb}$. It accounts for $10 \%$ of all

411 definitive differences and also shows low nucleotide diversity $(\pi=0.0004)$. These two sweep

412 regions on $\mathrm{X}$ show the lowest Tajima's $\mathrm{D}$ values in the genome outside of the $3 \mathrm{R}$ sweep region

413 (Xh vicinity $\mathrm{D}=-2.6 ;$ CYP9K1 vicinity $\mathrm{D}=-2.7$ ).

414 A final major genomic feature of AT concerns the $\mathrm{Y}$ chromosome. All AT individuals were

415 morphologically typed as female and confirmed as such due to coverage similarity between the $\mathrm{X}$

416 chromosome and autosomes. However, $94 \%$ of them showed high coverage across the majority of

417 the $\mathrm{Y}$ chromosome, except for the first $50 \mathrm{~kb}$ which includes the sex-determining region and sex-

418 determining gene $Y G 2$ (Figure 4C; Table 2). For most of the $\mathrm{Y}$ chromosome after $50 \mathrm{~kb}$, coverage

419 exceeded the autosomal and X-chromosome averages by over an order of magnitude, implying

420 that this sequence occurs repeatedly. This pattern is also observed in GOUNDRY, but not in $A$.

421 coluzzii from Tengrela (Table 2). The most likely explanation is that multiple copies of most of

422 the $\mathrm{Y}$ chromosome have merged with an autosome or the $\mathrm{X}$ chromosome in AT, consistent with

423 the highly repetitive and dynamic nature of the Anopheles Y (Hall et al., 2016).

424 Given the signal of inbreeding reported in GOUNDRY (Crawford et al. 2016), we 425 examined AT extensively for inbred individuals. There is no overall homozygote excess in AT.

426 The F coefficient of inbreeding is close to zero and slightly negative on average (mean $=-0.035$,

427 median $=-0.027)$. As in GOUNDRY, we observe large tracts of homozygosity in most individuals

428 (Figure 5A). However, regions of low heterozygosity are to be expected when overall nucleotide

429 diversity in the population is low (Figure 4B), so this is not necessarily a signal of inbreeding. 
430 Heterozygosity does not show a bimodal distribution (i.e. distinct regions of high or low

431 heterozygosity), but rather most low heterozygosity regions appear to be the tail end of a

432 continuous distribution (Figure 5B). Furthermore, fewer than $0.01 \%$ of variants are homozygous

433 doubletons, and these are not enriched in homozygosity tracts (5 doubletons are in tracts with mean

434 heterozygosity $\leq 1 \mathrm{e}-06$; expect $7.7 ; \mathrm{p}>0.1$ ). Finally, our haploid dataset demonstrated that our

435 demographic conclusions are robust to inbreeding. For example, in ADMIXTURE a two-

436 population model is still favored, separating AT from A. coluzzii, with GOUNDRY showing 78.5-

$43791.3 \%$ AT-like ancestry. Similarly, in dadi an admixed model is favored with GOUNDRY

438 showing $85.4 \%$ ancestry from AT (Supp Table 1).

439

$440 \quad$ A diagnostic protocol

441 In order to search for AT among additional samples, we developed a diagnostic protocol

442 based on amplicon sequencing. We first identified 50 diagnostic SNPs and small indels that

443 distinguish AT from Tengrela A. coluzzii and the Ag1000G samples (Supp Table 2; Supp Figure

444 7). We tested several pools of primer pairs and found that a pool of five primer pairs could be

445 jointly amplified and sequenced to sufficient coverage on an Illumina iSeq 100 . At these loci, the

446 diagnostic allele is fixed in AT, polymorphic in GOUNDRY (33-63\% frequency), and absent in

447 A. coluzzii and A. gambiae. We genotyped each locus by searching the reads for kmers overlapping

448 the target polymorphism. In a set of 10 AT specimens and 10 Tengrela A. coluzzii specimens, each

449 locus yielded an unambiguous genotype in every specimen (median number of read pairs per locus

450 per specimen $=7905$; mean $=9047$; range $=954$ to 25,180 ; Figure 6 ). Specifically, for all loci at

451 all AT specimens, more than $90 \%$ of read pairs had the AT allele, and for all loci at all A. coluzzii

452 specimens, more than $90 \%$ of read pairs had the A. coluzzii allele. The small numbers of incorrect 
453 reads could be due to index hopping among these jointly-sequenced samples (van der Valk, Vezzi,

454 Ormestad, Dalén, \& Guschanski, 2019) or low-level contamination. Coverage at the negative 455 control was lower but non-negligible ( 76 to 1460 read pairs per locus, mean $=411.6$ ), presumably

456 due to similar errors. However, for four out of five negative control loci, both alleles occurred in 457 more than $20 \%$ of read pairs. These results suggest that taxonomy can be confidently assigned and 458 false positives avoided if two filters are applied. First, specimens should be excluded if they have 459 fewer than $10 \%$ of the expected number of reads pairs (i.e. fewer than about 4000 read pairs for a 460 typical iSeq lane with 96 individuals). Second, for each specimen, a majority of loci should 461 implicate the same taxon with at least $90 \%$ of reads. Either one of these filters would exclude our 462 negative control.

We then used this pool of five primer pairs to amplify and sequence a novel set of 79 464 putative A. coluzzii specimens. All negative controls, and three real specimens, had fewer than 4654000 read pairs each and were discarded by the criteria outlined above. The remaining 76 466 specimens had acceptable coverage (median number of read pairs per locus per specimen $=1799$; 467 mean $=3466 ;$ range $=12$ to 25,357 ) and were all genotyped as unambiguously $A$. coluzzii 468 (maximum observed proportion of read pairs with AT allele: 2.6\%). Thus, we failed to detect AT 469 in this novel sample set.

\section{Discussion}

473 TENGRELA (AT). AT is related to but genetically distinct from sympatric A. coluzzii and shows 474 numerous fixed or nearly-fixed differences across the genome, as well as large difference in 
475 genomic architecture such as the $2 \mathrm{La}$ inversion polymorphism, the fixed $\mathrm{Xh}$ inversion, and the

476 presence of Y-chromosome-associated sequence in females. These differences are presumably

477 maintained by strong reproductive barriers. However, reproductive isolation is not complete, as

478 our demographic analysis indicated ongoing gene flow and a hybridization event within the last

479 thousand years (Figure 3). Such occasional gene flow is typical among nominal species of the $A$.

480 gambiae complex (Fontaine et al., 2015; Main et al., 2015). Lacking adequate phenotypic and

481 ecological data, we refrain from formally describing AT. However, it represents a unique lineage

482 and is not nested within A. coluzzii nor any other described species.

AT is most similar to GOUNDRY, another aberrant Anopheles population from Burkina

484 Faso. Our results indicate that GOUNDRY is an admixed population with most of its ancestry 485 originating from an AT-like lineage, but with substantial A. coluzzii ancestry as well. GOUNDRY 486 autosomes show 15-20\% A. coluzzii ancestry, while there is no evidence of $A$. coluzzii ancestry on 487 the $\mathrm{X}$ chromosome, which in the A. gambiae complex is typically more impervious to introgression 488 (Fontaine et al. 2015). Some GOUNDRY mitochondrial haplotypes are AT-like but most occur in 489 a distinct clade closer to A. coluzzii and A. gambiae (Figure 2B). There is no evidence for 490 reproductive barriers between AT and GOUNDRY; rather, GOUNDRY appears to be a relatively 491 new population recently diverged from the AT lineage via considerable introgression from $A$. 492 coluzzii. GOUNDRY is known to segregate for both S-form and M-form markers (Riehle et al. 493 2011), but the GOUNDRY genomes examined here resemble A. coluzzii (M-form) at diagnostic 494 markers (Table 2). Thus, it is possible that GOUNDRY has a more complex history involving 495 other ancestral lineages, beyond our ability to assess with the available data. The taxonomic status 496 of AT and GOUNDRY remains unresolved, but these two populations are not synonymous, and 497 AT appears to be more representative of the ancestral phylogenetic lineage. 
Our demographic model suggests that AT and A. coluzzii diverged 1.7 million generations ago, or 170,000 years ago assuming ten generations per year. This result depends on numerous

500 estimates, including mutation rate and the proportion of the genome genotyped accurately, and is 501 therefore imprecise. In general, we endeavored to be conservative in our estimate of differentiation 502 between AT and A. coluzzii. For example, we assumed no effect of purifying selection on the 503 variants used in demographic analysis, but if purifying selection has acted it would mean the true 504 time since divergence of AT and A. coluzzii is even greater than estimated here. Regardless, our 505 results suggest that cladogenesis predates the rise of agriculture in sub-Saharan Africa and was not 506 driven by adaptation to anthropogenically-disturbed habitats. The population sizes of both lineages 507 increased following this split, bolstered by cross-lineage migration. Then approximately 4,000 508 years ago AT decreased dramatically in population size while A. coluzzii increased further as is 509 well documented (Anopheles gambiae 1000 Genomes Consortium, 2017), leading to a modern $A$. 510 coluzzii population thousands of times larger than AT. If effective population size today 511 approximates census size, the relative rarity of AT could partially explain why it has not been 512 detected previously. Interestingly, the AT population crash occurred around 2000 BCE during the 513 advent of African agriculture (Shaw, 1972), which is hypothesized to have fostered the 514 diversification of the A. gambiae complex (Coluzzi et al., 2002; Crawford et al., 2016). Thus, the 515 decline of AT leading to its present-day rarity may have been driven by anthropogenic 516 modifications to habitat, which perhaps favored A. coluzzii instead. Our demographic model is 517 similar to the one previously suggested for comparing GOUNDRY and A. coluzzii (Crawford et 518 al., 2016). Relative to that model, our estimate of the split with A. coluzzii is slightly older, and 519 there are minor differences in population size changes and migration rates. By incorporating AT, 520 we reveal GOUNDRY's surprisingly recent origin as a distinct lineage. 
We know little about the ecology of AT. While GOUNDRY larvae have been reported in several sites across the hot, arid Sudano-Sahelian region of central Burkina Faso, AT in Tengrela occurs in the cooler, wetter, tropical savannah Sudanese climactic zone of southwestern Burkina

524 Faso. If GOUNDRY is derived from a local AT population, it would suggest that AT possesses a 525 broad tolerance for variable tropical climate. Alternatively, the A. coluzzii ancestry of GOUNDRY 526 may have permitted it to colonize more arid climates than AT can tolerate. We only observed AT 527 in 2011, the sole year when samples were collected in temporary puddles and not just rice paddies, 528 which suggests an ecological specificity. Although we cannot rule out immediate local decline or 529 extinction of AT between 2011 and 2012, such a dramatic change seems implausible, especially 530 when the absence of AT can be explained by our relatively small and ecologically limited sampling 531 scheme. Puddle-specificity of AT is also consistent with the known habitat of GOUNDRY (Riehle 532 et al., 2011). The putative selective sweep regions in AT may contain genes that convey unique 533 adaptive features to AT, but these remain to be characterized. Insecticide resistance alleles are 534 present in AT (Table 2), including at CYP9K1 which occurs within a putative selective sweep 535 region. Selection pressure from regular exposure to insecticide would imply that AT may 536 commonly occur in human-dominated habitats like the Tengrela village. We do not know if adults 537 are anthropophagous, or if they carry Plasmodium. Vectorial capacity is highly plausible for AT 538 given its close relationship to important malaria-transmitting taxa, so it will be critical to identify 539 and study AT adults to understand their potential impact on human health. Regardless of any direct vectorial capacity, cryptic Anopheles taxa have the potential to 541 stymie malaria control efforts in at least three ways. First, reproductive barriers can thwart efforts 542 to eliminate or modify the A. gambiae complex via gene drive (Marshall et al., 2019). A gene drive 543 targeting A. coluzzii will not necessarily spread to sympatric AT, which might even expand its 
544 population size in response to a population crash of A. coluzzii. Second, because reproductive

545 barriers are porous, adaptive genetic diversity maintained within AT can be shared with congeners

546 via introgression, enhancing their capacity to survive and evolve. Rare, semi-isolated taxa like AT

547 can thus serve as allelic reservoirs, facilitating adaptation to insecticides, gene drives, or even

548 climate change. For example, the 2La inversion is associated with adaptation to climate (Cheng et

549 al., 2012), and it shows heterozygote advantage in AT. Overdominance in AT could explain the

550 persistence of this polymorphism in a rare taxon with otherwise low nucleotide diversity. In

551 contrast, selection tends to eliminate one other the other 2La haplotype in A. coluzzii and A.

552 gambiae populations, so AT could be an important genetic reserve for these species if a previously

553 disfavored and purged allele becomes once again beneficial. Finally, misidentified cryptic taxa can

554 confound scientific studies and lead to incorrect inferences about Anopheles biology and

555 inaccurate predictions about disease epidemiology and the outcomes of vector control. For

556 example, the A. gambiae species complex first came to light following confusing discordances in

557 insecticide resistance phenotype between field and captive mosquito populations (Davidson 1956).

558 Captive mating experiments subsequently demonstrated the discordance was due to the inability

559 to discern A. gambiae from another morphologically identical member of the complex, $A$.

560 arabiensis (Davidson 1956, Davidson \& Jackson, 1962). More recently, Gildenhard et al. (2019)

561 noted a striking difference in TEP1 allele frequencies between two populations of A. coluzzii in

562 Burkina Faso, which was attributed to ecologically-varying selection. While this is a very plausible

563 and potentially accurate explanation, the results could also be explained by misidentified AT

564 within the samples, as AT has a very different TEP1 profile from A. coluzzii (Figure 4A, Table 2).

565 Since AT and A. coluzzii appear similar at standard diagnostic loci (Table 2), this is just one of

566 many studies on A. coluzzii that could be potentially confounded by AT. 
It will be crucial to correctly identify Anopheles taxa in order to draw accurate conclusions

568 that can inform disease control policy. Our amplicon-based diagnostic protocol (Figure 6; Supp

569 Table 2) provides a clear methodology for identifying AT. The pool of five primer pairs can be

570 amplified and sequenced jointly, yielding high accuracy. For further genotyping as needed, we

571 provide primer pair sequences for a total of 50 diagnostic polymorphisms (Supp Table 2; Supp

572 Figure 7), though not all primers have been empirically validated. Future work could improve upon

573 this methodology, perhaps via a PCR and electrophoresis method to target these polymorphisms

574 without sequencing. An important lesson from AT is that critical diversity can be missed if only a

575 small number of diagnostic markers are assessed, so we do not advocate a protocol based on any

576 one locus alone. We encourage Anopheles biologists to genotype existing DNA samples,

577 especially of putative $A$. coluzzii from Burkina Faso and adjacent countries, and to seek AT in

578 future surveys. As AT has only been found as larvae from a single site in a single year, we cannot

579 fully characterize it biologically without additional observations. AT will probably not be the last

580 cryptic taxon discovered within this extraordinarily diverse clade. Mapping these intertwined

581 lineages across Africa will be an essential ongoing task with an inestimable impact on human

582 health.

584 Acknowledgements

585 Our thanks to the residents of Tengrela where this study was conducted, and especially volunteer

586 field workers from this village. We are grateful to the field team from CNRFP for helping with

587 mosquito collection. We also thank Sanjay Nagi, Patricia Pignatelli, Natalie Lissenden, Nithya

588 Swaminathan, Akanksha Khorgade, and Tim Farrell for assistance with sample collection, 
molecular preparation, and/or bioinformatics. Data interpretation was aided by helpful 590 commentary from Angela Early, Stephen Schaffner, Aimee Taylor, Seth Redmond, and others.

591 Mosquito collections in Burkina Faso were supported by EC FP7 Project grant no: 265660

592 "AvecNet" and Wellcome Trust Collaborative Award (200222/Z/15/Z). This project has been

593 funded in whole or in part with Federal funds from the National Institute of Allergy and Infectious

594 Diseases, National Institutes of Health, Department of Health and Human Services, under Grant

595 Number U19AI1 10818 to the Broad Institute.

\section{References}

Alexander, D.H., Novembre, J., \& Lange, K. (2009). Fast model-based estimation of ancestry in unrelated individuals. Genome Research, 19, 1655-1664. doi: 10.1101/gr.094052.109

Anopheles gambiae 1000 Genomes Consortium. (2017). Genetic diversity of the African malaria vector Anopheles gambiae. Nature, 552, 96-100. doi: 10.1038/nature24995 Ayala, D. (2019) A new species in the major malaria vector complex sheds light on reticulated species evolution. Scientific Reports, 9, 14753. doi: 10.1038/s41598-019-49065-5.

Bhatt, S., Weiss, D.J., Cameron, E., Bisanzio, D., Mappin, B., Dalrymple, U., ... Gething, P.W. (2015). The effect of malaria control on Plasmodium falciparum in Africa between 2000 and 2015. Nature, 526, 207-211. doi: 10.1038/nature15535

Chang, C.C., Chow, C.C., Tellier, L.C., Vattikuti. S., Purcell, S.M., Lee, J.J. (2015) Secondgeneration PLINK: rising to the challenge of larger and richer datasets. Gigascience 4, 7. doi: 10.1186/s13742-015-0047-8

Cheng, C., White, B.J., Kamdem, C., Mockaitis, K., Costantini, C., Hahn, M.W., \& Besansky, N.J. (2012). Ecological genomics of Anopheles gambiae along a latitudinal cline: a populationresequencing approach. Genetics, 190, 1417-1432. doi: 10.1534/genetics.111.137794

Clarkson, C.S., Weetman, D., Essandoh, J., Yawson, A.E., Maslen, G., Manske, M., ... Donnelly, M.J. (2014). Adaptive introgression between Anopheles sibling species eliminates a major genomic island but not reproductive isolation. Nature Communications, 5, 4248. doi: 10.1038/ncomms5248 
625 Coetzee, M., Hunt, R.H., Wilkerson, R., della Torre, A., Coulibaly, M.B., \& Besansky N.J. (2013).

626 Anopheles coluzzii and Anopheles amharicus, new members of the Anopheles gambiae complex.

627 Zootaxa, 3619, 246-274.

628

629

630

631

632

633

634

635

636

637

638

639

640

641

642

643

644

645

646

647

648

649

650

651

652

653

654

655

656

657

658

659

660

661

662

663

664

665

666

667

668 669
Coluzzi, M., Sabatini, A., della Torre, A., Di Deco, M.A., \& Petrarca, V. (2002). A polytene chromosome analysis of the Anopheles gambiae species complex. Science, 298, 1415-1418.

Crawford, J.E., Riehle, M.M., Guelbeogo, W.M., Gneme, A., Sagnon, N., Vernick, K.D., ... Lazzaro, B.P. (2015). Reticulate speciation and barriers to introgression in the Anopheles gambiae species complex. Genome Biology and Evolution, 7, 3116-3131. doi: 10.1093/gbe/evv203

Crawford, J.E., Riehle, M.M., Markianos, K., Bischoff, E., Guelbeogo, W.M., Gneme, A., ... Lazzaro, B.P. (2016). Evolution of GOUNDRY, a cryptic subgroup of Anopheles gambiae s.l., and its impact on susceptibility to Plasmodium infection. Molecular Ecology, 25, 1494-1510. doi: $10.1111 /$ mec. 13572

Davidson, G. (1956). Insecticide resistance in Anopheles gambiae Giles. Nature, 178, 705-706. doi: $10.1038 / 178705 \mathrm{a} 0$

Davidson, G., \& Jackson, C.E. (1962). Incipient speciation in Anopheles gambiae Giles. Bulletin of the World Health Organization, 27: 303-305

Donnelly, M.J., Corbel, V., Weetman, D., Wilding, C.S., Williamson, M.S., \& Black, W.C. (2009). Does kdr genotype predict insecticide-resistance phenotype in mosquitoes? Trends in Parasitology, 25: 213-219. doi: 10.1016/j.pt.2009.02.007

Du, W., Awolola, T.S., Howell, P., Koekemoer, L.L., Brooke, B.D., Benedict, M.Q., ... Zheng, L. (2005). Independent mutations in the $R d l$ locus confer dieldrin resistance to Anopheles gambiae and An. arabiensis. Insect Molecular Biology, 14, 179-183.

Fanello, C., Santolamazza, F., \& della Torre, A. (2002). Simultaneous identification of species and molecular forms of the Anopheles gambiae complex by PCR-RFLP. Medical and Veterinary Entomology, 16, 461-464.

Fontaine, M.C., Pease, J.B., Steele, A., Waterhouse, R.M., Neafsey, D.E., Sharakhov, I.V., ... Besansky, N.J. (2015). Mosquito genomics. Extensive introgression in a malaria vector species complex revealed by phylogenomics. Science, 347,1258524 . doi: 10.1126/science.1258524

Gildenhard, M., Rono, E.K., Diarra, A., Boissière, A., Bascunan, P., Carrillo-Bustamante, P., ... Levashina, E.A. (2019). Mosquito microevolution drives Plasmodium falciparum dynamics. Nature Microbiology, 4, 941-947. doi: 10.1038/s41564-019-0414-9

Gillies MT, \& De Meillon B. (1968). The Anophelinae of Africa South of the Sahara (Ethiopian Zoogeographical Region) (2 ${ }^{\text {nd }}$ ed.). Johannesburg: South African Institute for Medical Research. 
Giraldo-Calderón, G.I., Emrich, S.J., MacCallum, R.M., Maslen, G., Dialynas, E., Topalis, P., ... Lawson, D. (2015). VectorBase: an updated bioinformatics resource for invertebrate vectors and other organisms related with human diseases. Nucleic Acids Research, 43, D707-13. doi: 10.1093/nar/gku1117

Gutenkunst, R.N., Hernandez, R.D., Williamson, S.H., \& Bustamante, C.D. (2009). Inferring the joint demographic history of multiple populations from multidimensional SNP frequency data. PLoS Genetics, 5, e1000695. doi: 10.1371/journal.pgen.1000695

Greppi, C., Laursen, W.J., Budelli, G., Chang, E.C., Daniels, A.M., van Giesen, L., ... Garrity, P.A. (2020). Mosquito heat seeking is driven by an ancestral cooling receptor. Science, 367, 681684.

Hall, A.B., Papathanos, P.A., Sharma, A., Cheng, C., Akbari, O.S., Assour, L., ... Besansky, N.J. (2016). Radical remodeling of the Y chromosome in a recent radiation of malaria mosquitoes. Proceedings of the National Academy of Sciences of the United States of America, 113, E2114 2123. doi: $10.1073 /$ pnas. 1525164113

Huang, Y., Guo, Q., Sun, X., Zhang, C., Xu, N., Xu, Y., ... Shen, B. (2018). Culex pipiens pallens cuticular protein CPLCG5 participates in pyrethroid resistance by forming a rigid matrix. Parasites \& Vectors, 11, 6. doi: 10.1186/s13071-017-2567-9

Keightley, P.D., Ness, R.W., Halligan, D.L., \& Haddrill, P.R. (2014). Estimation of the spontaneous mutation rate per nucleotide site in a Drosophila melanogaster full-sib family. Genetics, 196, 313-320. doi: 10.1534/genetics.113.158758

Holt, R.A., Subramanian, G.M., Halpern, A., Sutton, G.G., Charlab, R., Nusskern, D.R., ... Hoffman, S.L. (2002). The genome sequence of the malaria mosquito Anopheles gambiae. Science, 298, 129-149.

Lee, Y., Marsden, C.D., Norris, L.C., Collier, T.C., Main, B.J., Fofana, A., ... Lanzaro, G.C. (2013). Spatiotemporal dynamics of gene flow and hybrid fitness between the $\mathrm{M}$ and $\mathrm{S}$ forms of the malaria mosquito, Anopheles gambiae. Proceedings of the National Academy of Sciences of the United States of America, 110, 19854-19859. doi: 10.1073/pnas.1316851110

Leffler, E.M., Bullaughey, K., Matute, D.R., Meyer, W.K., Ségurel, L., Venkat, A., ... Przeworski, M. (2012). Revisiting an old riddle: what determines genetic diversity levels within species? PLoS Biology, 10, e1001388.

Li, H., \& Durbin, R. (2009). Fast and accurate short read alignment with Burrows-Wheeler Transform. Bioinformatics, 25, 1754-1760.

Li, H. (2011) A statistical framework for SNP calling, mutation discovery, association mapping and population genetical parameter estimation from sequencing data. Bioinformatics, 27, 2987- 
Main, B.J., Lee, Y., Collier, T.C., Norris, L.C., Brisco, K., Fofana, A., ... Lanzaro, G.C. (2015)

717 Complex genome evolution in Anopheles coluzzii associated with increased insecticide usage in 718 Mali. Molecular Ecology, 24, 5145-5157. doi: 10.1111/mec.13382

Marshall, J.M., Raban, R.R., Kandul, N.P., Edula, J.R., León, T.M., \& Akbari, O.S. (2019). Winning the tug-of-war between effector gene design and pathogen evolution in vector population replacement strategies. Frontiers in Genetics, 10, 1072. doi: 10.3389/fgene.2019.01072

McKenna, A., Hanna, M., Banks, E., Sivachenko, A., Cibulskis, K., Kernytsky, A., ... DePristo, M.A. (2010). The Genome Analysis Toolkit: a MapReduce framework for analyzing nextgeneration DNA sequencing data. Genome Research, 20, 1297-1303. doi: 10.1101/gr.107524.110

Neafsey, D.E., Lawniczak, M.K.N., Park, D.J., Redmond, S.N., Coulibaly, M.B., Traoré, S.F., ... Muskavitch, M.A.T. (2010). SNP genotyping defines complex gene-flow boundaries among African malaria vector mosquitoes. Science, 330, 514-517. doi: 10.1126/science.1193036

Norris, L.C., Main, B.J., Lee, Y., Collier, T.C., Fofana, A., Cornel, A.J., \& Lanzaro G.C. (2015). Adaptive introgression in an African malaria mosquito coincident with the increased usage of insecticide-treated bed nets. Proceedings of the National Academy of Sciences of the United States of America, 112, 815-820. doi: 10.1073/pnas.1418892112

Nkya, T.E., Poupardin, R., Laporte, F., Akhouayri, I., Mosha, F., Magesa, S., ... David, J.P. (2014). Impact of agriculture on the selection of insecticide resistance in the malaria vector Anopheles gambiae: a multigenerational study in controlled conditions. Parasites \& Vectors, 7, 480. doi: 10.1186/s13071-014-0480-z

Pickrell, J.K., \& Pritchard, J.K. (2012) Inference of population splits and mixtures from genomewide allele frequency data. PLoS Genetics, 8, e1002967. doi: 10.1371/journal.pgen.1002967

R Core Team. (2019). R: A language and environment for statistical computing. R Foundation for Statistical Computing, Vienna, Austria. https://www.R-project.org/

Ranson, H., \& Lissenden, N. (2016). Insecticide resistance in African Anopheles mosquitoes: a worsening situation that needs urgent action to maintain malaria control. Trends in Parasitology, 32, 187-196. doi: 10.1016/j.pt.2015.11.010

Riehle, M.M., Guelbeogo, W.M., Gneme, A., Eiglmeier, K., Holm, I., Bischoff, E., ... Vernick, K.D. (2011). A cryptic subgroup of Anopheles gambiae is highly susceptible to human malaria parasites. Science, 331, 596-598. doi: 10.1126/science.1196759

Rottschaefer, S.M., Riehle, M.M., Coulibaly, B., Sacko, M., Niaré, O., Morlais, I., ... Lazzaro, B.P. (2011). Exceptional diversity, maintenance of polymorphism, and recent directional selection on the APL1 malaria resistance genes of Anopheles gambiae. PLoS Biology, 9, e1000600. 
Santolamazza, F., Mancini, E., Simard, F., Qi, Y., Tu, Z., \& della Torre A. (2008). Insertion polymorphisms of SINE200 retrotransposons within speciation islands of Anopheles gambiae molecular forms. Malaria Journal, 7, 163. doi: 10.1186/1475-2875-7-163

Scott, J.A., Brogdon, W.G., \& Collins, F.H. (1993). Identification of single specimens of the Anopheles gambiae complex by the polymerase chain reaction. The American Journal of Tropical Medicine and Hygiene, 49, 520-529.

Sharakhova, M.V., Hammond, M.P., Lobo, N.F., Krzywinski, J., Unger, M.F., Hillenmeyer, M.E., ... Collins, F.H. (2007). Update of the Anopheles gambiae PEST genome assembly. Genome Biology, 8, R5.

Shaw, T. (1972). Early agriculture in Africa. Journal of the Historical Society of Nigeria, 6, 143191.

Stajich, J.E., Hahn, M.W. (2005). Disentangling the effects of demography and selection in human history. Molecular Biology and Evolution, 22, 63-73.

Stamatakis, A. (2006). RAxML-VI-HPC: maximum likelihood-based phylogenetic analyses with thousands of taxa and mixed models. Bioinformatics, 22, 2688-2690.

[dataset] Tennessen, J.A., Ingham, V.A., Toé, K.H., Guelbéogo' W.M., Sagnon N., Kuzma R., ... Neafsey, D.E.; 2020; Whole-genome sequencing of Anopheles mosquitoes from Tengrela, Burkina Faso; NCBI SRA; BioProject ID PRJNA639055

van der Valk, T., Vezzi, F., Ormestad, M., Dalén, L., \& Guschanski, K. (2019). Index hopping on the Illumina HiseqX platform and its consequences for ancient DNA studies. Molecular Ecology Resources, doi: 10.1111/1755-0998.13009

White, B.J., Hahn, M.W., Pombi, M., Cassone, B.J., Lobo, N.F., Simard, F., Besansky, N.J. (2007). Localization of candidate regions maintaining a common polymorphic inversion (2La) in Anopheles gambiae. PLoS Genetics, 3, e217.

White, B.J., Lawniczak, M.K., Cheng, C., Coulibaly, M.B., Wilson, M.D., Sagnon, N., ... Besansky, N.J. (2011). Adaptive divergence between incipient species of Anopheles gambiae increases resistance to Plasmodium. Proceedings of the National Academy of Sciences of the United States of America, 108, 244-249. doi: 10.1073/pnas.1013648108

You, F.M., Huo, N., Gu, Y.Q., Luo, M.C., Ma, Y., Hane, D., .. Anderson, O.D. (2008). BatchPrimer3: a high throughput web application for PCR and sequencing primer design. BMC Bioinformatics, 9, 253. doi: 10.1186/1471-2105-9-253

\section{Data Accessibility}


803 Raw Illumina reads from whole-genome sequencing have been deposited in NCBI SRA, 804 Bioproject ID PRJNA639055, at https://www.ncbi.nlm.nih.gov/sra.

805

\section{Author contributions}

807 JAT performed all data analyses and wrote the paper with the assistance of all co-authors. RK 808 headed the development and testing of the amplicon panel. VAI, KHT, WMG, NS, and HR 809 provided samples and assisted with data interpretation. DEN oversaw the project and assisted with 810 data interpretation.

811 
813 Table 1. Samples examined.

\begin{tabular}{|c|c|c|c|c|}
\hline Data Source & Year & Sampling & Taxon & $\mathbf{N}$ \\
\hline \multirow{3}{*}{$\begin{array}{l}\text { This study: } \\
\text { whole genome } \\
\text { sequencing }\end{array}$} & \multirow[t]{3}{*}{$2011(\mathrm{~N}=72)$} & \multirow{3}{*}{$\begin{array}{l}\text { Tengrela } \\
\text { (southwestern } \\
\text { Burkina Faso): } \\
\text { puddles and/or } \\
\text { rice paddies }\end{array}$} & AT & 51 \\
\hline & & & A. coluzzii & 20 \\
\hline & & & Unidentified & 1 \\
\hline \multirow{3}{*}{$\begin{array}{l}\text { This study: } \\
\text { whole genome } \\
\text { sequencing }\end{array}$} & \multirow{3}{*}{$\begin{array}{l}2012(\mathrm{~N}=71) \\
2015(\mathrm{~N}=72) \\
2016(\mathrm{~N}=72)\end{array}$} & \multirow{3}{*}{$\begin{array}{l}\text { Tengrela } \\
\text { (southwestern } \\
\text { Burkina Faso): } \\
\text { rice paddies }\end{array}$} & AT & 0 \\
\hline & & & A. coluzzii & 208 \\
\hline & & & Unidentified & 7 \\
\hline \multirow{3}{*}{$\begin{array}{l}\text { This study: } \\
\text { amplicon } \\
\text { genotyping }\end{array}$} & \multirow[t]{3}{*}{$\begin{array}{l}\text { Various / } \\
\text { unknown }\end{array}$} & \multirow{3}{*}{$\begin{array}{l}\text { Bounouna or } \\
\text { Nafona } \\
\text { (southwestern } \\
\text { Burkina Faso) } \\
\text { or unknown }\end{array}$} & AT & 0 \\
\hline & & & A. coluzzii & 79 \\
\hline & & & Unidentified & 3 \\
\hline \multirow[t]{2}{*}{$\begin{array}{l}\text { Crawford et } \\
\text { al., } 2016 \text { : }\end{array}$} & \multirow[t]{2}{*}{$2007-2008$} & \multirow[t]{2}{*}{$\begin{array}{l}\text { Central } \\
\text { Burkina Faso }\end{array}$} & GOUNDRY & 12 \\
\hline & & & A. coluzzii & 10 \\
\hline
\end{tabular}




\begin{tabular}{|l|l|l|l|l|}
\hline whole genome & & & & \\
sequencing & & & & \\
\hline
\end{tabular}

34 


\begin{tabular}{|c|c|c|c|c|c|c|c|}
\hline Locus & Description & Chromosome & Position & Allele & $\begin{array}{l}\text { AT } \\
\text { Frequency }\end{array}$ & $\begin{array}{l}\text { GOUNDRY } \\
\text { Frequency }\end{array}$ & $\begin{array}{l}\text { Tengrela Anopheles } \\
\text { coluzzii Frequency }\end{array}$ \\
\hline \multirow[t]{2}{*}{$2 \mathrm{La}$} & \multirow[t]{2}{*}{$\begin{array}{l}22 \quad \mathrm{Mb} \\
\text { inversion }\end{array}$} & \multirow[t]{2}{*}{$2 \mathrm{~L}$} & \multirow[t]{2}{*}{$\begin{array}{l}20000000- \\
42000000\end{array}$} & $2 \mathrm{~L}+{ }^{\mathrm{a}}$ (haplotype) & $48 \%$ & $70 \%$ & $3 \%$ \\
\hline & & & & $\begin{array}{l}2 \mathrm{~L}^{\mathrm{a}} 2 \mathrm{~L}+^{\mathrm{a}} \text { (heterozygous } \\
\text { genotype) }\end{array}$ & $\begin{array}{l}73 \% \\
\text { (expect } \\
50 \% ; \chi^{2}= \\
10.5 ; \quad P= \\
0.001)\end{array}$ & $\begin{array}{l}31 \% \text { (expect } \\
42 \% ; \quad \chi^{2}= \\
36.2 ; \quad P< \\
0.0001)\end{array}$ & $5 \%($ expect $5 \%)$ \\
\hline
\end{tabular}




\begin{tabular}{|c|c|c|c|c|c|c|c|}
\hline Y-linked & $\begin{array}{l}\text { Y- } \\
\text { chromosome } \\
\text { sequence in } \\
\text { PEST, } \\
\text { excluding } \\
\text { sex- } \\
\text { determining } \\
\text { gene }\end{array}$ & $\mathrm{Y}$ & $\begin{array}{l}50000- \\
230000\end{array}$ & Y sequence present & $94 \%$ & $100 \%$ & $3 \%$ \\
\hline$R d l$ & $\begin{array}{l}\text { Insecticide } \\
\text { resistance }\end{array}$ & $2 \mathrm{~L}$ & 25429235 & SER (resistant) & $32 \%$ & $58 \%$ & $\begin{array}{l}68 \% \text { in } 2011-2012 \\
38 \% \text { in } 2015-2016\end{array}$ \\
\hline$K d r$ & $\begin{array}{l}\text { Insecticide } \\
\text { resistance }\end{array}$ & $2 \mathrm{~L}$ & 2422652 & PHE (resistant) & $51 \%$ & $46 \%$ & $78 \%$ \\
\hline СУР9K1 & $\begin{array}{l}\text { Insecticide } \\
\text { resistance }\end{array}$ & $\mathrm{X}$ & 15242000 & cyp-II (resistant?) & $100 \%$ & $67 \%$ & $11 \%$ \\
\hline
\end{tabular}




\begin{tabular}{|c|c|c|c|c|c|c|c|}
\hline TEPI & $\begin{array}{l}\text { Plasmodium } \\
\text { resistance }\end{array}$ & $3 \mathrm{~L}$ & 11205000 & $\mathrm{R}$ (protective) & $0 \%$ & $12 \%$ & $98 \%$ \\
\hline APL1A & $\begin{array}{l}\text { Plasmodium } \\
\text { resistance }\end{array}$ & $2 \mathrm{~L}$ & 41271000 & APL1A $^{2}$ (protective) & $15 \%$ & $17 \%$ & $80 \%$ \\
\hline $\mathrm{Xh}$ & $\begin{array}{l}1.5 \quad \mathrm{Mb} \\
\text { inversion }\end{array}$ & $\mathrm{X}$ & $\begin{array}{l}8470000- \\
10100000 \\
\text { (example } \\
\text { SNP at } \\
9361641)\end{array}$ & Non-reference & $100 \%$ & $100 \%$ & $0 \%$ \\
\hline $\begin{array}{l}\text { Sweep } \\
\text { region }\end{array}$ & $\begin{array}{l}\text { Unknown } \\
\text { phenotypic } \\
\text { effect }\end{array}$ & $3 R$ & $\begin{array}{l}8900000- \\
13300000 \\
\text { (example } \\
\text { SNP at } \\
13081325)\end{array}$ & $\mathrm{T}$ (non-reference) & $100 \%$ & $88 \%$ & $0 \%$ \\
\hline
\end{tabular}




\begin{tabular}{|c|c|c|c|c|c|c|c|}
\hline rDNA IGS & $\begin{array}{l}\text { Intergenic } \\
\text { region of } \\
\text { ribosomal } \\
\text { DNA } \\
\text { (multiple } \\
\text { copies) }\end{array}$ & $\begin{array}{l}\text { UNK } \\
\text { (sequence } \\
\text { from Scott et } \\
\text { al., 1993) }\end{array}$ & $580-581$ & $\begin{array}{l}\text { Hhal cut site (S-form } \\
\text { specific; Fanello et al., } \\
\text { 2002) }\end{array}$ & $0 \%$ & $8 \%$ & $0 \%$ \\
\hline \multirow[t]{2}{*}{$\begin{array}{l}\mathrm{M} / \mathrm{S} \\
\text { diagnostic } \\
\text { SNPs }\end{array}$} & \multirow[t]{2}{*}{$\begin{array}{l}\text { Divergence } \\
\text { island SNPs } \\
\text { (Lee et al., } \\
\text { 2013) }\end{array}$} & $2 \mathrm{~L}$ & $\begin{array}{l}209536, \\
1274353, \\
2430786, \\
2430915, \\
2431005\end{array}$ & M-form & $46-51 \%$ & $54-58 \%$ & $22-28 \%$ \\
\hline & & $3 \mathrm{~L}$ & $\begin{array}{l}296897, \\
387877, \\
413944\end{array}$ & M-form & $99-100 \%$ & $100 \%$ & $100 \%$ \\
\hline
\end{tabular}




\begin{tabular}{|c|c|c|c|c|c|c|c|}
\hline & & $\mathrm{X}$ & $\begin{array}{l}20015634, \\
22105429, \\
22105860, \\
22497157,2 \\
2750432, \\
22750572, \\
22944682\end{array}$ & M-form & $99-100 \%$ & $96 \%$ & $100 \%$ \\
\hline \multirow[t]{2}{*}{ S200 X6.1 } & $\begin{array}{l}230 \mathrm{bp} \\
\text { diagnostic } \\
\text { indel }\end{array}$ & $\mathrm{X}$ & 22951000 & $\mathrm{M}$-form insertion present & $100 \%$ & $100 \%$ & $100 \%$ \\
\hline & $\begin{array}{l}\text { SNP within } \\
\text { insertion }\end{array}$ & $\mathrm{X}$ & 22951586 & $\mathrm{~T}$ (non-reference) & $100 \%$ & $100 \%$ & $1 \%$ \\
\hline
\end{tabular}




\section{Figure Legends}

819 Figure 1. Genetic distinctiveness of AT. (A) In a PCA plot with Tengrela, GOUNDRY, and 820 Ag1000G individuals, AT occurs as a distinct cluster close to GOUNDRY. Variants were chosen based on segregation in our data and may show ascertainment bias affecting the relationships of individuals within Ag1000G; the salient result is how AT and Tengrela A. coluzzii relate to these other individuals (B) AT remains distinct in a PCA after combining Tengrela samples with GOUNDRY and the A. coluzzii samples collected alongside GOUNDRY. To control for differences between studies, all reads were trimmed to the same length, and then alignment and genotyping were performed jointly.

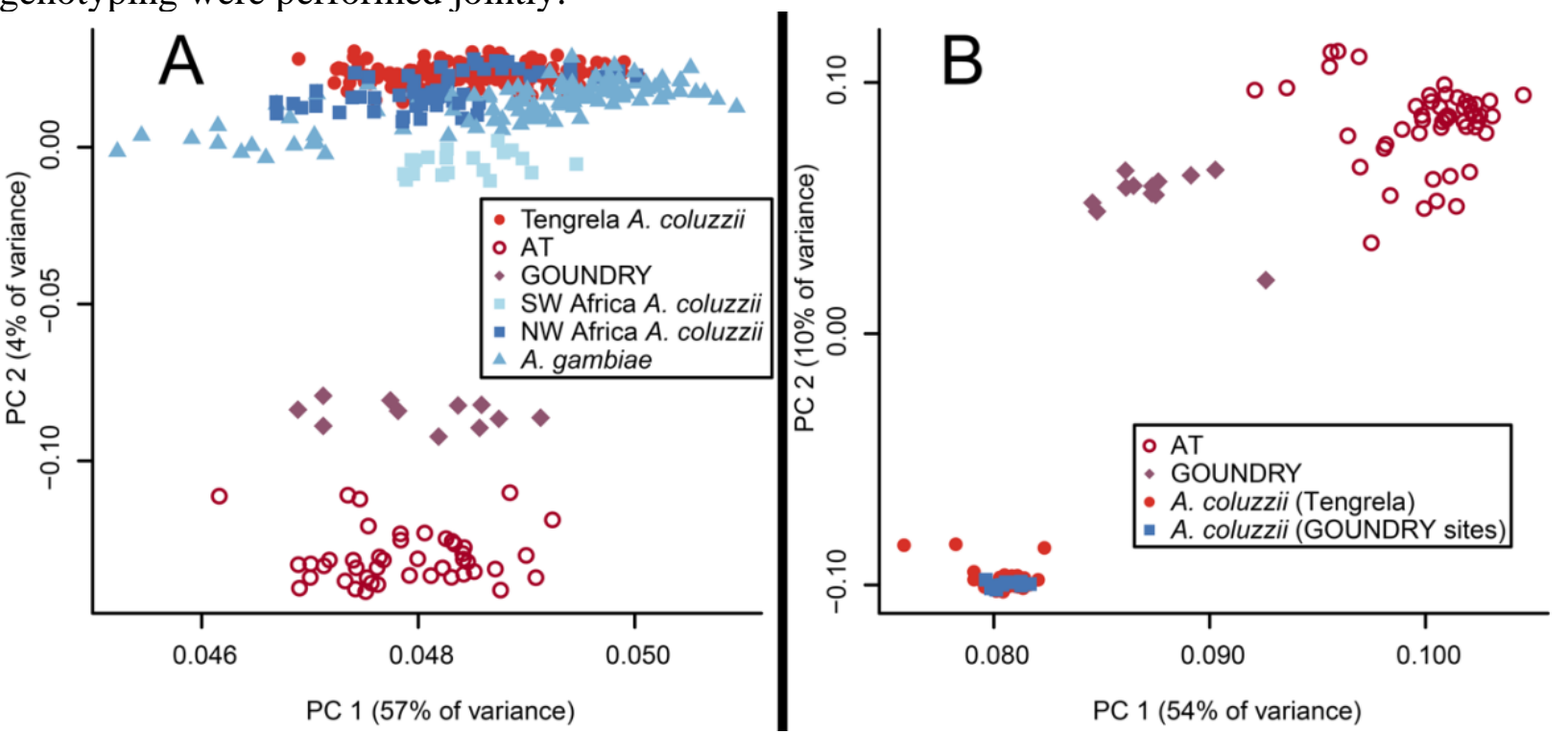

Figure 2. (A) Most common phylogenetic topology among sections of AT genome and nominal species of the A. gambiae complex. Numbers at branches are not bootstraps, but the percentage of $100 \mathrm{~kb}$ windows that support each clade (above branches: entire genome; below branches: $\mathrm{X}$ chromosome only). AT is sister to A. coluzzii across $42.1 \%$ of the genome $(55.7 \%$ of the X chromosome), more often than to any other species, and $95.5 \%$ of the genome (100.0\% of the X chromosome) supports a clade with AT, A. coluzzii and A. gambiae to the exclusion of the other species. (B) The mitochondrial DNA phylogeny shows that AT shares a single haplotype that occupies a unique branch close to the A. gambiae PEST reference genome. GOUNDRY samples occur near AT or near A. coluzzii and A. gambiae samples from Tengrela and elsewhere in Burkina Faso (BF), consistent with an admixed origin. 


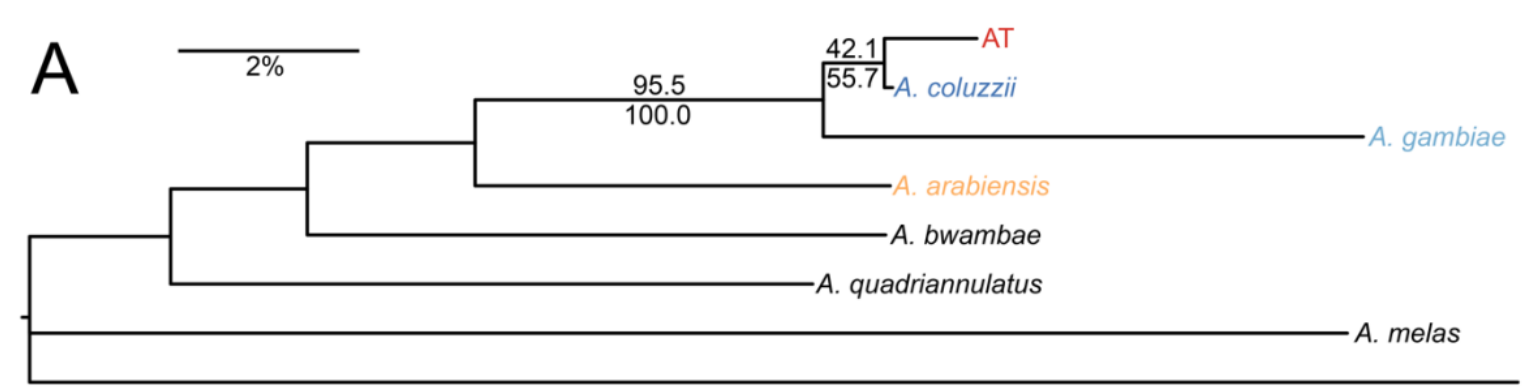

A. merus

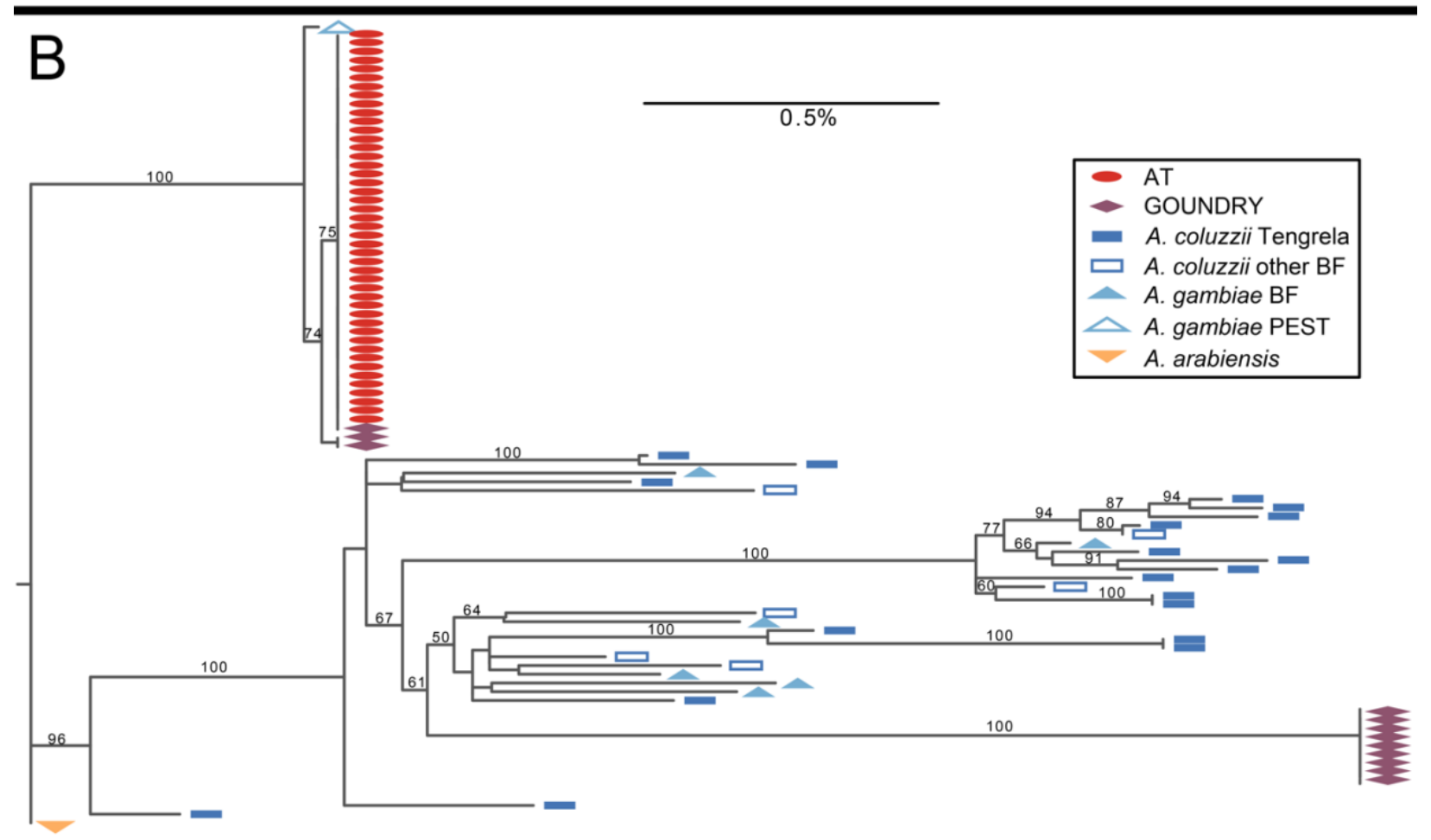

Figure 3: Relationships between AT, GOUNDRY, and A. coluzzii autosomes using jointly-called genotypes. (A) Analysis with ADMIXTURE suggests two ancestral populations, closely 843 approximated by contemporary AT and A. coluzzii, with GOUNDRY showing ancestry from both. 844 (B) Analysis with dadi corroborates this model, with an AT/A. coluzzii split over one million 845 generations ago, followed by ongoing gene flow and a recent admixed origin of GOUNDRY. 846 Population sizes (heights of colored bars) and migration rates (widths of arrows) vary across three 847 time periods (demarcated with dotted lines). (C) Analysis with TreeMix shows GOUNDRY as 848 sister to AT but with in-migration from A. coluzzii. 


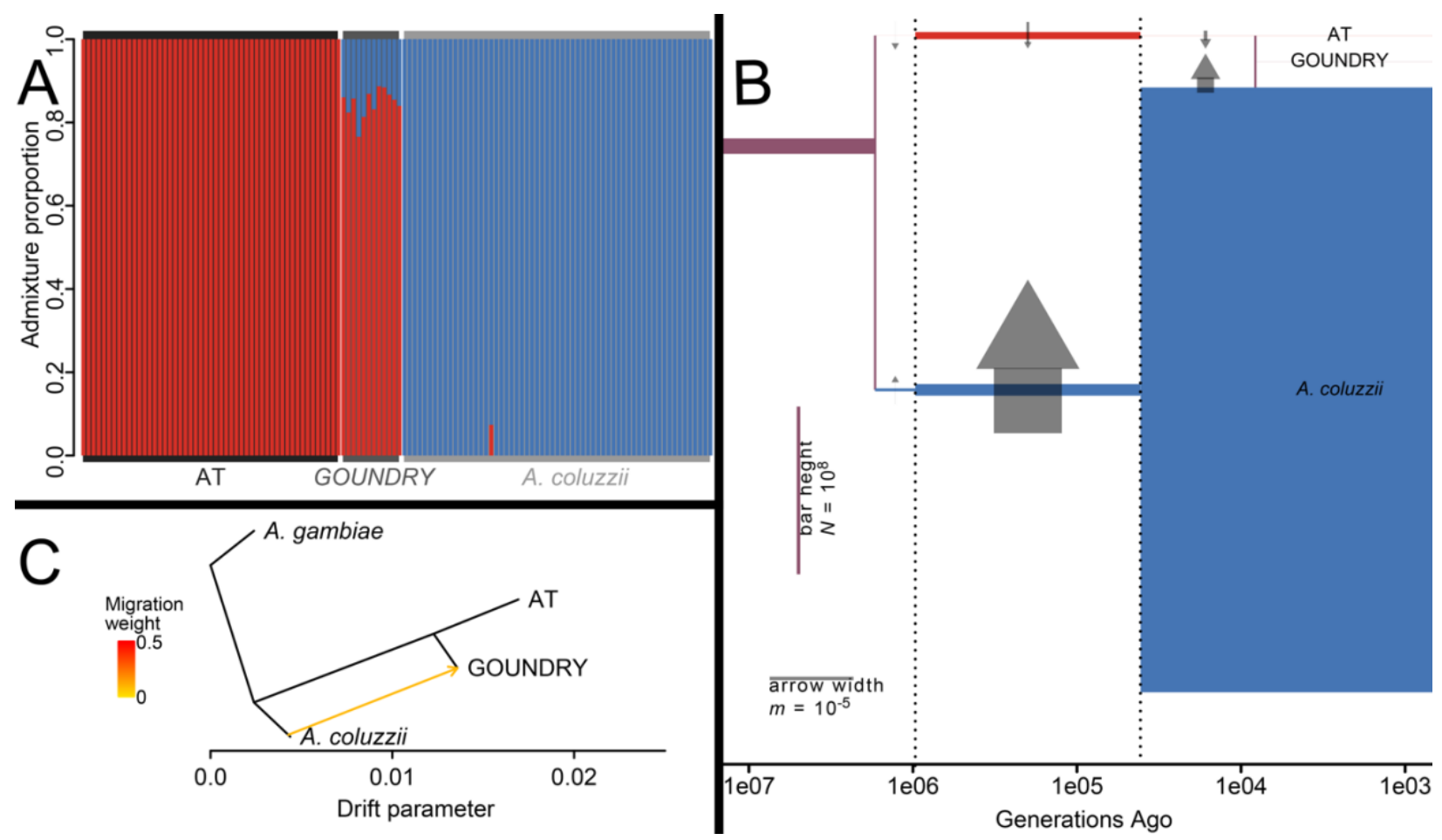

Figure 4. Unique genomic characteristics of AT. (A) FST across the genome between AT and Tengrela A. coluzzii. Tens of thousands of variants distributed across the genome are highly divergent between these taxa ( $\mathrm{F}_{\mathrm{ST}}>0.8$; orange dots at top), while over a thousand sites, concentrated in several clusters, are fixed or nearly so ("definitive differences", FST >0.99; red dots at top). Average FST in $100 \mathrm{~kb}$ windows is more modest (blue lines), but three regions stand out representing the $2 \mathrm{La}$ inversion, TEP1, and $C Y P 9 K 1$. (B) Nucleotide diversity $(\pi)$, intertaxon divergence (Dxy), and site frequency spectra (Tajima's D) in AT and A. coluzzii. Nucleotide diversity is low in AT except at the 2La inversion. Tajima's D is mostly positive in AT, but three regions show low Tajima's D, low $\pi$, and many high-Fst sites (as shown in A), suggesting selective sweeps. (C) In most AT females, read coverage along most of the reference Y chromosome substantially exceeds the X/autosomal average. Coverage is negligible around sex-determining gene $Y G 2$. A. coluzzii females, in contrast, typically show negligible coverage across the entire $\mathrm{Y}$ except for a few repetitive sections. 


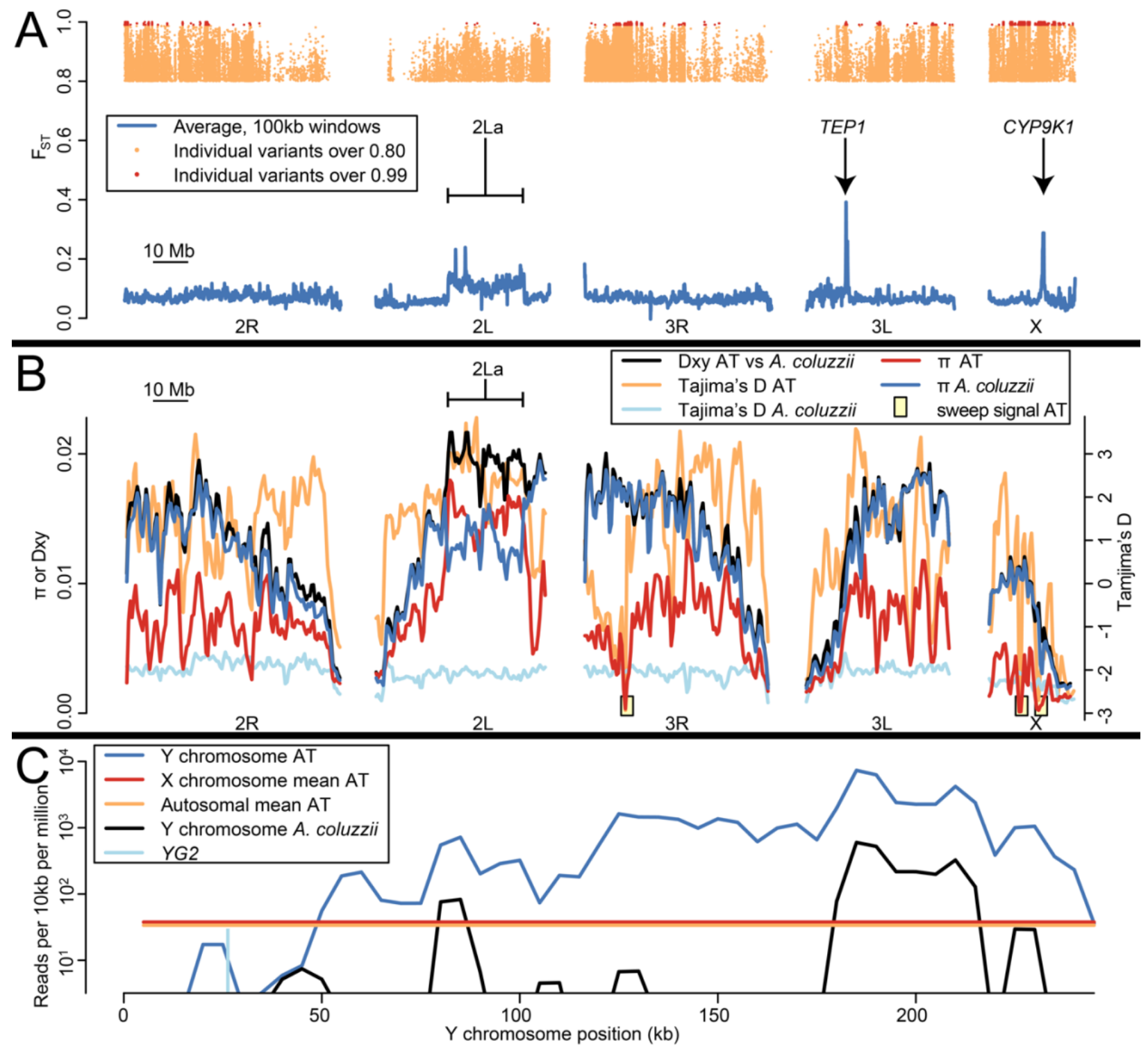

Figure 5. Regions of low heterozygosity in AT. (A) Heterozygosity $(H)$ in blocks of $1 \mathrm{Mb}$ across the genome for all 51 AT individuals. There are many long homozygosity tracts (red) in most individuals, but these are consistent with the relatively low genetic diversity observed across the population, except in the 2La inversion. (B) Histogram of the heterozygosity blocks depicted in A, based on number of heterozygous sites. Heterozygosity shows a relatively smooth distribution with only a slight uptick for homozygous blocks ( 0 or 1 heterozygous sites), indicating little evidence 872 for inbreeding driving homozygosity. 


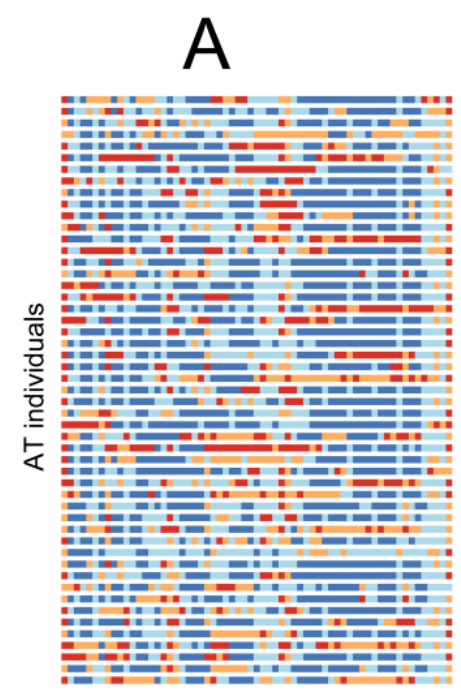

$2 \mathrm{R}$

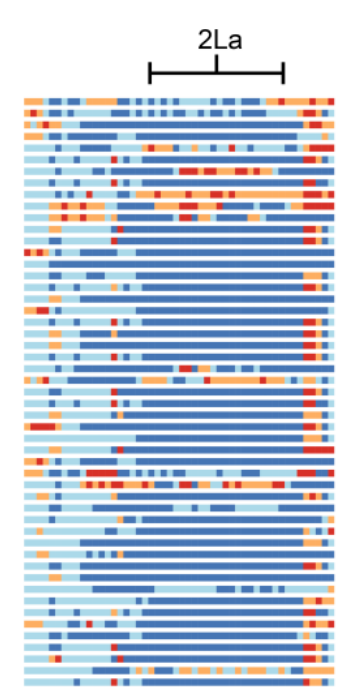

$2 \mathrm{~L}$

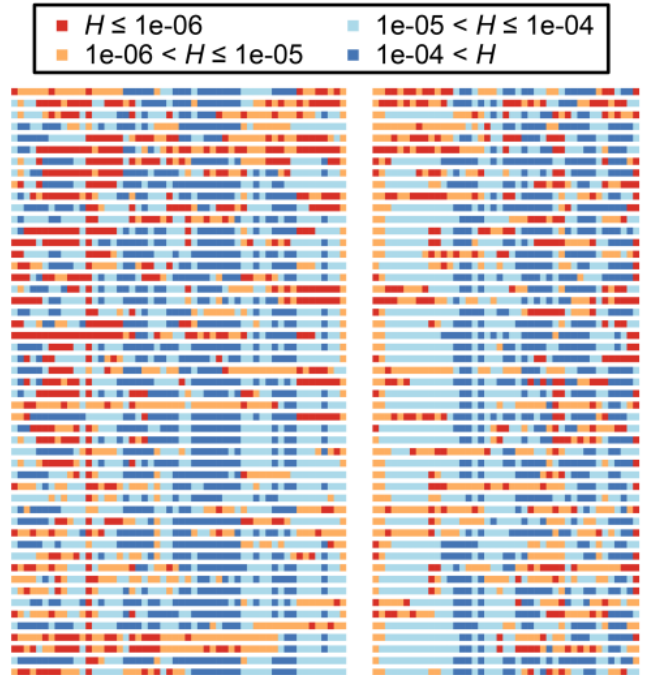

3L

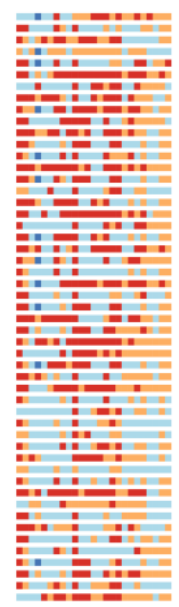

X

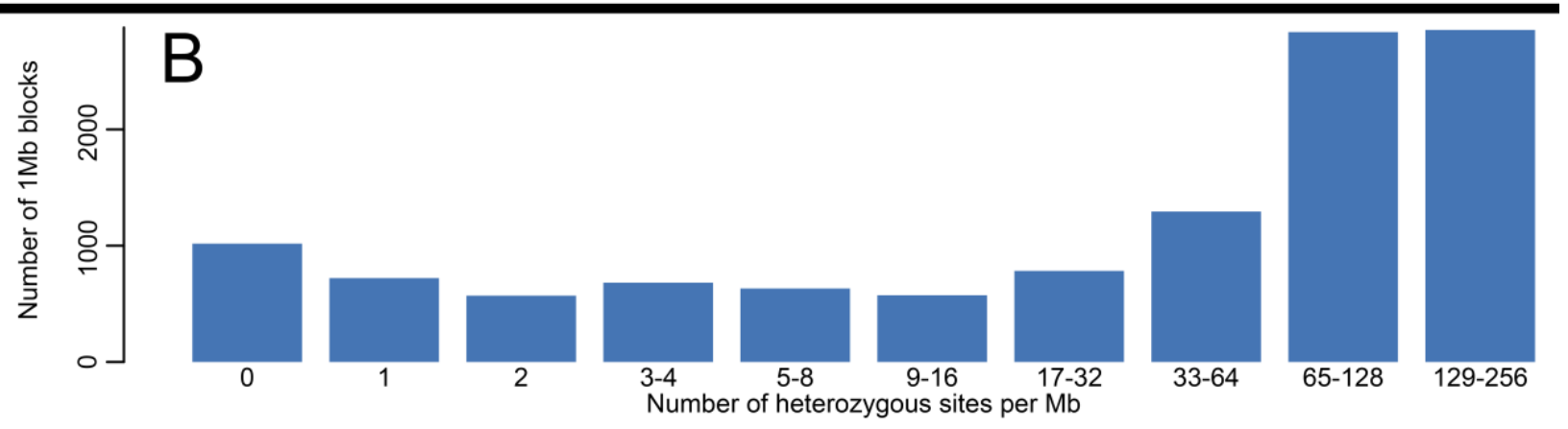

873

874

875

876

877

878

879

880

881

882

Figure 6. Distinguishing AT from A. coluzzii using amplicon genotyping. A pool of five primer pairs were selected that amplify five polymorphisms diagnostic for AT, and jointly amplified PCR products were sequenced (10 AT, $10 \mathrm{~A}$. coluzzii, and one negative control). For each marker, the vast majority of read pairs are consistent with the known taxonomic category as inferred from whole genome sequencing, allowing for unambiguous identification. A small number of read pairs were erroneously assigned to the negative control ("neg"), indicating that a conservative test should exclude any individual with unusually low coverage and/or intermediate frequencies of both alleles.

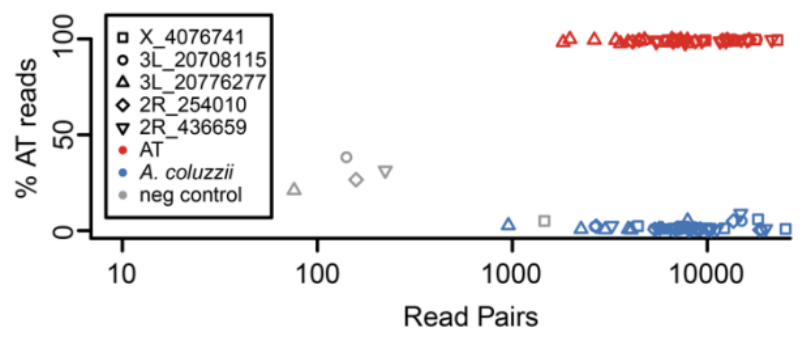

